\title{
CONCEPTUal Designs for IR OPTICS AT C0
}

\author{
JOHN A. JOHNSTONE \\ FERMILAB
}

JUNE $15^{\text {th }}, 2000$ 


\section{CONTENTS}

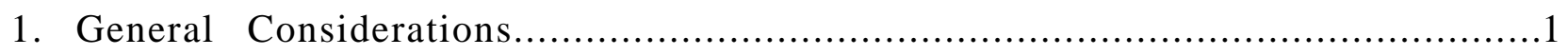

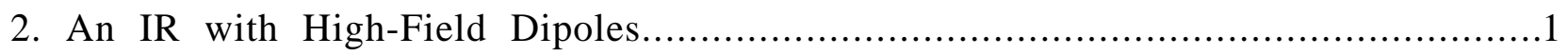

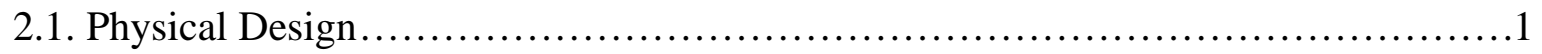

2.1.1. Quadrupoles .........................................................

2.1.2. Dipoles \& Separators ..................................................... 3

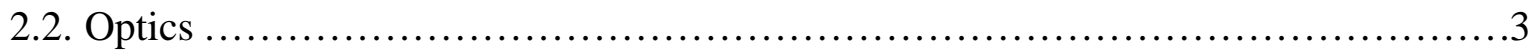

2.2.1. B0, C0, \& D0 Low $\beta *$ Squeezes ...................................... 4

2.2.1.1. $\beta^{*}=4.00 @ \mathrm{C} 0-\left(\beta \mathrm{x}^{*}, \beta \mathrm{y}\right)=(1.61,1.74) @ \mathrm{~B} 0 \& \mathrm{D} 0 \ldots . . . .5$

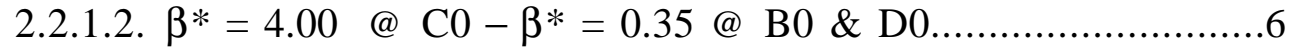

2.2.1.3. $\beta^{*}=0.50 @ \mathrm{C} 0-\left(\beta \mathrm{x}^{*}, \beta \mathrm{y}^{*}\right)=(1.61,1.74) @ \mathrm{~B} 0 \& \mathrm{D} 0 \ldots . . .7$

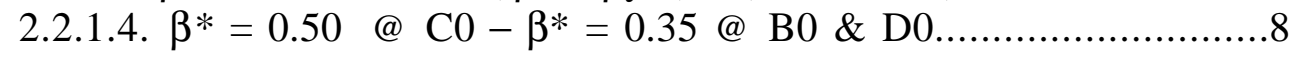

2.3. Beam Separation ............................................................... 10

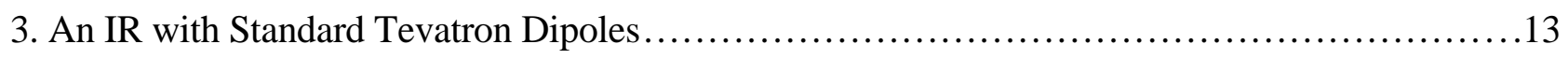

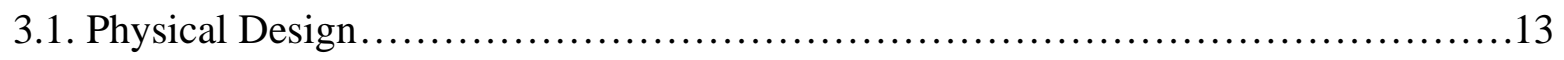

3.1.1. Quadrupoles ..................................................... 13

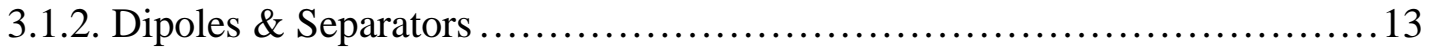

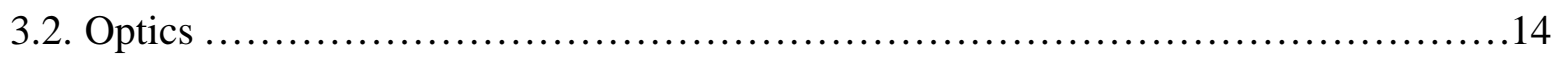

3.3. Beam Separation ................................................................ 15

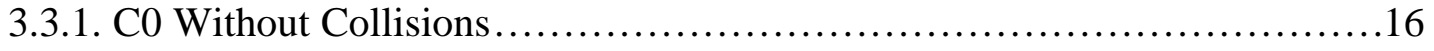

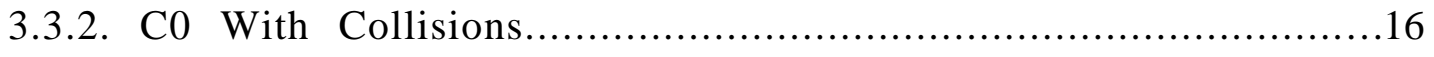

3.4. Collisions at B0, C0, \& D0 ................................................

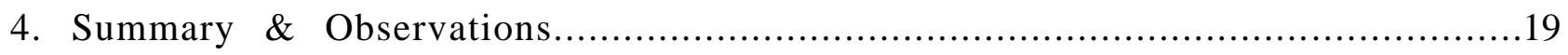




\section{General Considerations}

Given the advanced state of operational plans for Run IIb (132 nsec bunch spacing) a new C0 Interaction Region (IR) insertion should be capable of operating in a manner that does not impact nominal Run IIb Tevatron parameters. This implies creating an entirely localized insertion - one which is completely transparent to the rest of the machine. This constraint has several important design implications, some of which are pointed out below.

- An IR design similar to that employed at CDF \& D0 is unacceptable as a C0 candidate. The addition of such a (single) low- $\beta$ region to the machine raises the tune by a half-integer in each plane, moving them far from the standard operating point and smack onto the 21.0 integer resonance. The nominal (fractional) tunes are most elegantly maintained by adding 2 low- $\beta$ 's locally in each plane, thereby boosting the machine tunes by a full integer.

- The B0 \& D0 IR's are not optically-isolated entities. Progression through the low- $\beta$ squeeze involves adjusting, not only the main IR quadrupoles, but also the tune quad strings distributed around the ring. The result is that the lattice functions at any point in the ring, and the phase advances across any section of the ring, are not fixed quantities, but vary through the squeeze sequence. The C0 IR optics must be sufficiently flexible to track these elusive matching conditions.

- With collisions only at B0 \& D0 the unit transfer matrix added by the C0 insert ensures that the incoming \& outgoing helices are automatically matched into the established Run IIb values. To maintain this match with collisions at all 3 IP's, however, requires that additional separators be added in the arcs. Space for these separators can only be generated by replacing standard Tevatron arc dipoles with new magnets having enhanced field strengths.

In subsequent sections two variations of an interaction region design are presented. The first of these, which incorporates stronger dipoles, fulfills all the ideal design criteria outlined above. The result is a truly independent $3^{\text {rd }}$ Tevatron IR capable of supporting simultaneous collisions at all 3 IP's. The second, stripped-down, version includes neither stronger dipoles nor new arc separators. While this insert is also optically transparent to the machine, useful collisions can only occur at B0 \& D0, or just $\mathrm{C} 0$, but not all three. The restriction to standard-strength dipoles also results in a significant reduction of the space available for a detector.

\section{AN IR WITH HIGH-FIELD DIPOLES}

\subsection{Physical Design}

\subsubsection{Quadrupoles}

Both the series \& independent IR quad circuits are illustrated below. The new magnets required fall into 3 gradient ranges. There are LHC-like magnets operating in the vicinity of $180 \mathrm{~T} / \mathrm{m}$. This is substantially less than the $>220 \mathrm{~T} / \mathrm{m}$ LHC design, but the gradients are limited in this application by the Tevatron $4.2 \mathrm{~K}$ cryogenics. High-field $140 \mathrm{~T} / \mathrm{m}$ quadrupoles, modeled like existing magnets installed at the other 2 IR's, are also used. And there are high gradient $(\geq 60 \mathrm{~T} / \mathrm{m})$ correction spools which, again, are comparable to those at CDF \& D0. 


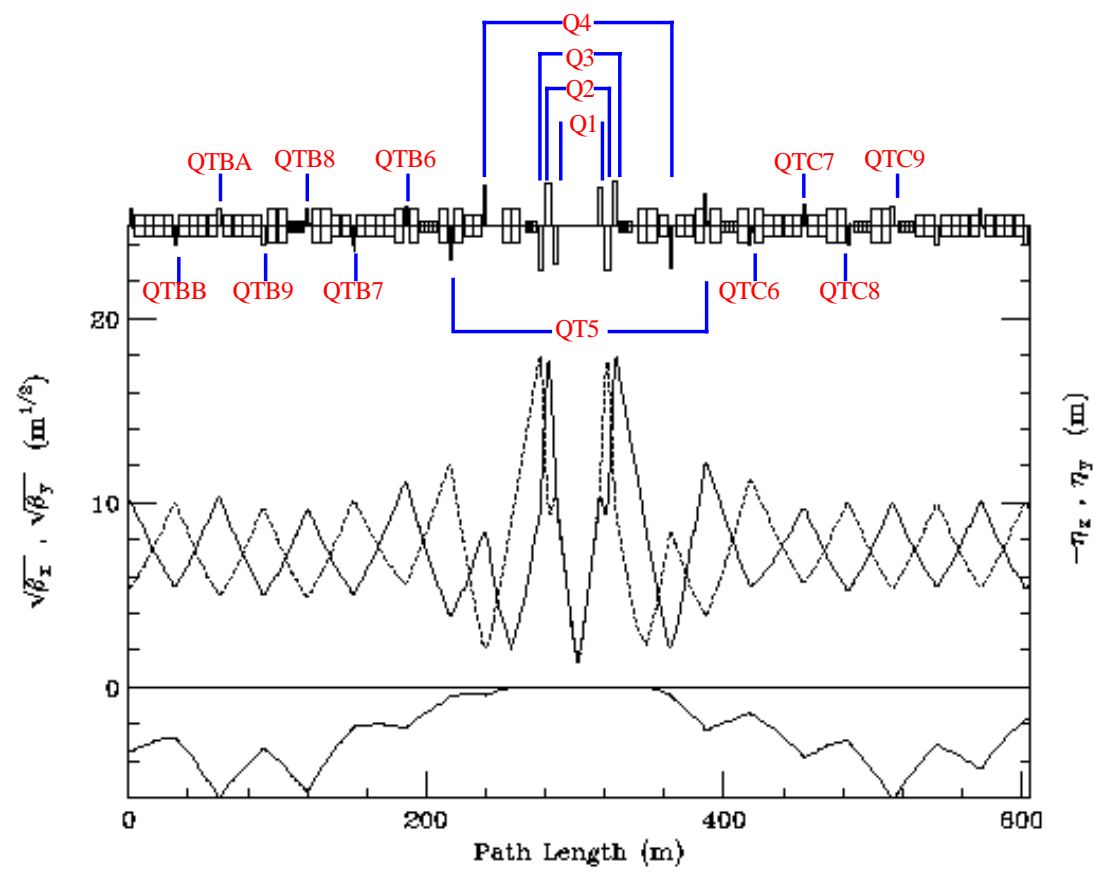

Composition of the quadrupole circuits is described below, with the indicated lengths being magnetic lengths:

$\begin{array}{lll}\text { Q1 } & : 93^{\prime \prime} & \text { LHC } \\ \text { Q2 } & : 160^{\prime \prime} & \text { LHC } \\ \text { Q3 } & : 93^{\prime \prime} & \text { LHC }\end{array}$

Unlike the triplets at B0 \& D0, the inner \& outer quadrupole circuits (Q1 \& Q3 ) of the final focus in this model are powered separately.
Q4
[B48 / C12 ] : 66"
LHC

The Q4 quads are accompanied by short (56") spools containing just dipole correctors \& BPM's in both planes.

$$
\begin{array}{lllr}
\text { Q5 \& Q5T [ B47 / C13 ] } & \text { : } 55.19 " & 140 \mathrm{~T} / \mathrm{m} \\
& : 25^{\prime \prime} & 60 \mathrm{~T} / \mathrm{m}
\end{array}
$$

The regular 66" arc quads plus their spools at B47 \& C13 are replaced by high field 55" magnets, operating at a constant $136 \mathrm{~T} / \mathrm{m}$, plus a series connection of tunable strong correctors for focusing adjustments.

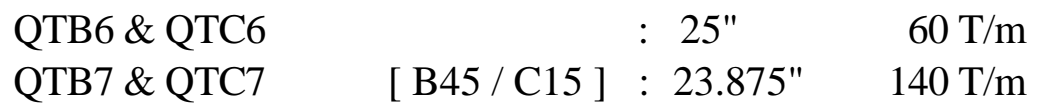

At B45 \& C15 the Tevatron 66" arc quads and their short spools are replaced by (existing) 32" magnets powered on the main buss plus very strong, independently-powered correctors like those currently installed at the A47, B13, C47, \& D13 locations ${ }^{1}$.

1 This configuration actually increases (by 5") the space available for power feeds. 
All the remaining trim quad spools - QTB8 $\rightarrow$ QTBB, and QTC8 \& QTC9 - are of the 25", strong, $60 \mathrm{~T} / \mathrm{m}$ variety.

Non-standard separations appear between some of the insertion's inner arc quadrupoles. Between the B48 \& B47 [C12 \& C13] quadrupoles space is reduced from 4 to 3 dipoles, whereas between B46 \& B45 [C14 \& C15] separation increases by 1 dipole slot length. Extensive simulations have shown this configuration contributes markedly to the robustness of the IR's optical versatility.

Trim quads are allocated in a lop-sided configuration, with 2 more installed in the upstream end of the insert. In B-sector it is possible to extend insert elements a good distance back into the arc before interfering with Run IIb operations. Not so in C-sector. The 4 vertical separators at C17 are integral components of Run IIb controls and, therefore, define the downstream insert boundary.

\subsubsection{Dipoles \& Separators}

A total of 30 standard Tevatron dipoles are replaced by 20 high-field dipoles. This has two substantial benefits ${ }^{2}$. First, additional longitudinal space for the detector is generated by installing extra strength bends in the vicinity of the IP. In the current design there is $>13 \mathrm{~m}$ of free space each side of the IP. Second, by replacing 12 standard dipoles with 8 stronger ones in the B- \& C-sector arcs, sufficient space is created between B43 \& B44 [C16 \& C17] to install 4 separators, and another 4 between B46 \& B47 [C13 \& C14].

There are 3 separators each side of the IP, immediately outboard of the triplets, for controlling beam position at the IP. New arc separators are necessary both for angle control at the IP and for matching the incoming to outgoing helix. Without these the 3 IR's can not operate independently.

\subsection{Optics}

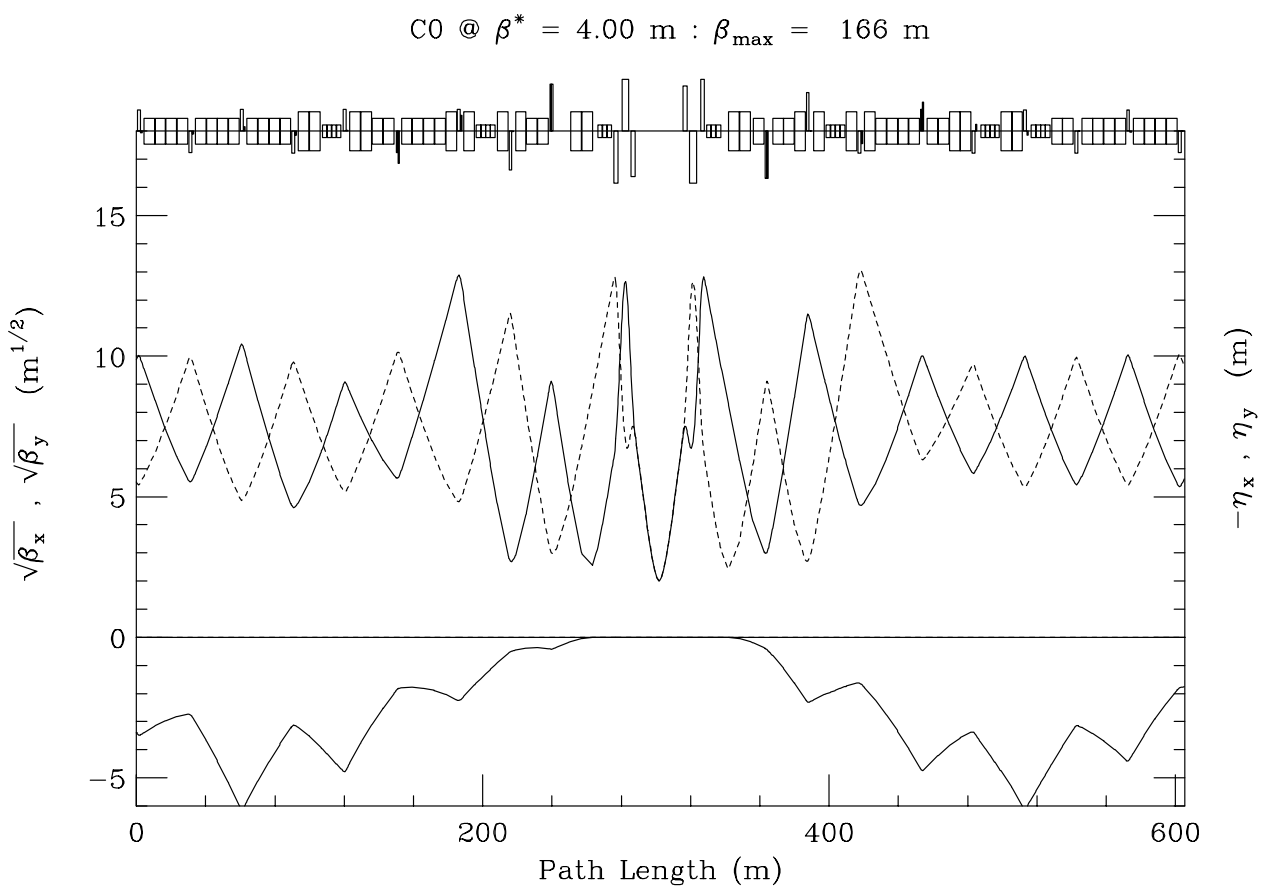

2 A 3rd, lesser, bonus is that the Tevatron orbit shrinks by about $11 \mathrm{~mm}$ - helping somewhat to alleviate the infamous $4 \mathrm{~cm}$ circumference mismatch between the Main Injector \& Tevatron. 
In the injection lattice, shown above, $\beta^{*}=4.00 \mathrm{~m}$ results in a $\beta$ max of only $166 \mathrm{~m}$ in the triplets. This is considerably less than the $>240 \mathrm{~m}$ of the B0 \& D0 injection lattices, and not too much larger than the $120 \mathrm{~m}$ of a standard Collins insert.

Tevatron Collider experience suggests that the smallest realistic $\beta^{*}$ attainable is limited by the good-field aperture and, therefore, $\beta$ max in the low $-\beta$ triplets, rather than by any gradient limitations of the IR quads. In the current model the Q1 magnets at C0 are roughly 20' farther from the IP than the corresponding ones at B0 \& D0. As a result, $\beta$ max is considerably larger at $\mathrm{C} 0$ for any given value of $\beta^{*}$. With $\beta^{*}=50 \mathrm{~cm}, \beta$ max has already grown to $1230 \mathrm{~m}$. This is somewhat larger $(\sim 12 \%)$ than the $\beta \max$ for $\beta^{*}=35 \mathrm{~cm}$ at the other IP's, but is probably still acceptable.

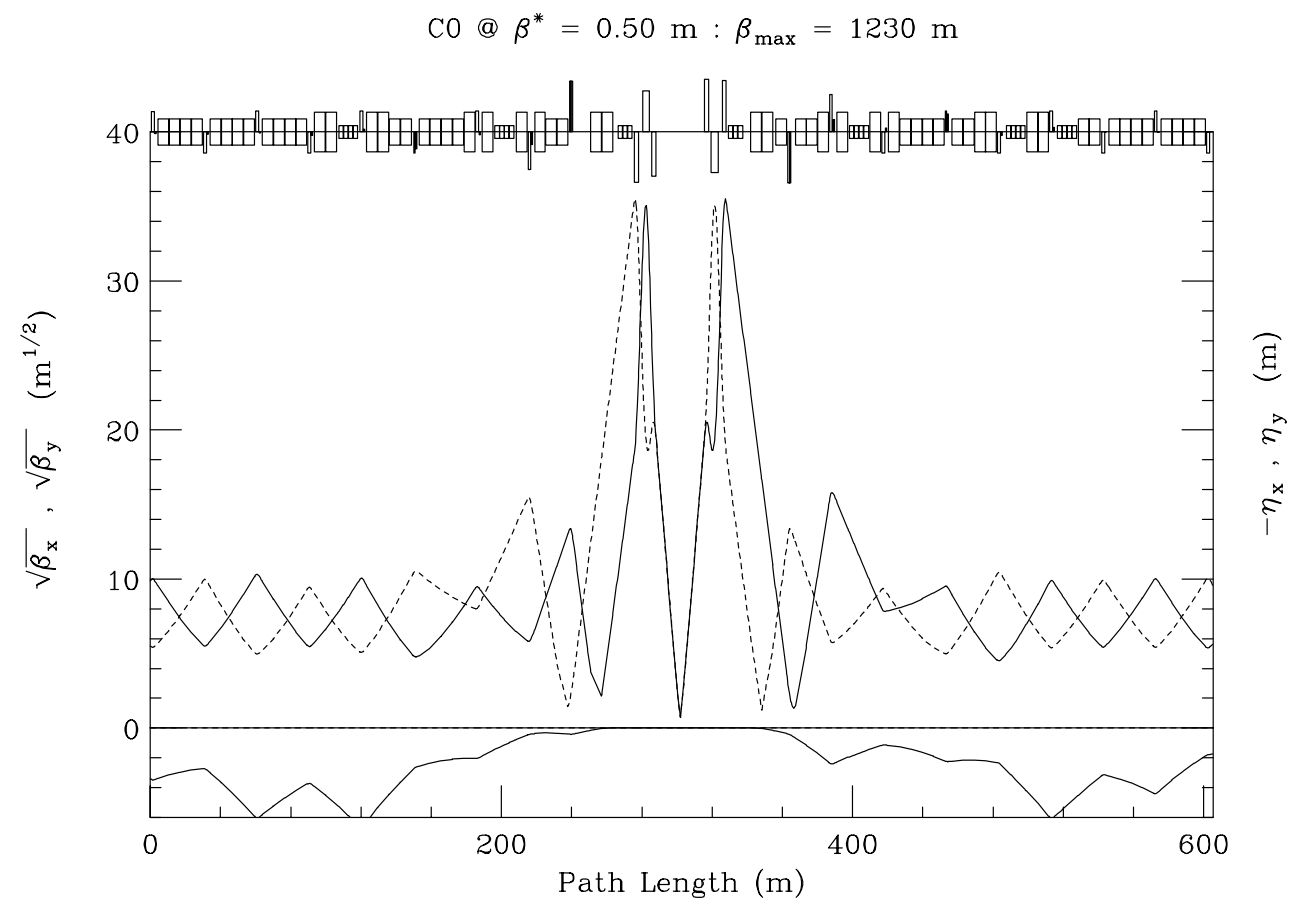

\subsubsection{BO, C0, \& DO Low $-\beta^{*}$ Squeezes}

There are 16 optical constraints the insertion must satisfy. The 6 incoming Twiss parameters are matched at the IP to $\beta_{\mathrm{X}}{ }^{*}=\beta_{\mathrm{y}}{ }^{*} \equiv \beta^{*}, \alpha_{\mathrm{X}}{ }^{*}=\alpha_{\mathrm{y}}{ }^{*} \equiv 0, \eta^{*} \equiv 0, \eta^{\prime}{ }^{*} \equiv 0$, and then matched back into the nominal arc values at the downstream end of the insert (at C17). The fractional Run IIb phase shifts, $\Delta \mu_{\mathrm{X}}$ and $\Delta \mu_{\mathrm{y}}$, are preserved across the insert. The final constraint imposed is the insistence that $\beta_{\max }(x)=\beta_{\max }(y)$ in the triplets on each side of the IP. While this last restriction isn't really crucial, it is the best choice, minimizing the consumption of aperture in the low- $\beta$ quads.

In the design discussed here, every stage of the squeeze from $\beta^{*}=4.00 \rightarrow 0.50 \mathrm{~m}$ at $\mathrm{C} 0$ can match exactly to any step in the Injection $\rightarrow \beta^{*}=0.35 \mathrm{~m}$ squeeze at B0 \& D0. The following pages illustrate the lattice functions \& tabulate the $\mathrm{C} 0$ quadrupole gradients corresponding to the extremes of this operational matrix:
(1) $\beta^{*}=4.00 @ \mathrm{C} 0-\left(\beta_{\mathrm{X}}^{*}, \beta_{\mathrm{y}}^{*}\right)=(1.61,1.74) @ \mathrm{~B} 0 \& \mathrm{D} 0$
(2) $\beta^{*}=4.00 @ \mathrm{C} 0-\beta^{*}=0.35 @ \mathrm{~B} 0$ \& D0 
PAC 00

(3) $\beta^{*}=0.50 @ \mathrm{C} 0-\left(\beta_{\mathrm{x}}{ }^{*}, \beta_{\mathrm{y}}{ }^{*}\right)=(1.61,1.74) @ \mathrm{~B} 0 \& \mathrm{D} 0$

(4) $\beta^{*}=0.50 @ \mathrm{C} 0-\beta^{*}=0.35 @ \mathrm{~B} 0 \& \mathrm{D} 0$

All gradients in the following tables reflect $1 \mathrm{TeV} / \mathrm{c}$ operations. Highlighted entries indicate magnets that change polarity at some point during the squeezes.

2.2.1.1. $\beta^{*}=4.00 @ \mathrm{C} 0-\left(\beta \mathrm{x}^{*}, \beta \mathrm{y}\right)=(1.61,1.74) @ \mathrm{~B} 0 \& \mathrm{D} 0$

$\mathrm{CO} @ \beta^{*}=4.00 \mathrm{~m}: \mathrm{BO} \& \mathrm{DO} @$ INJECTION

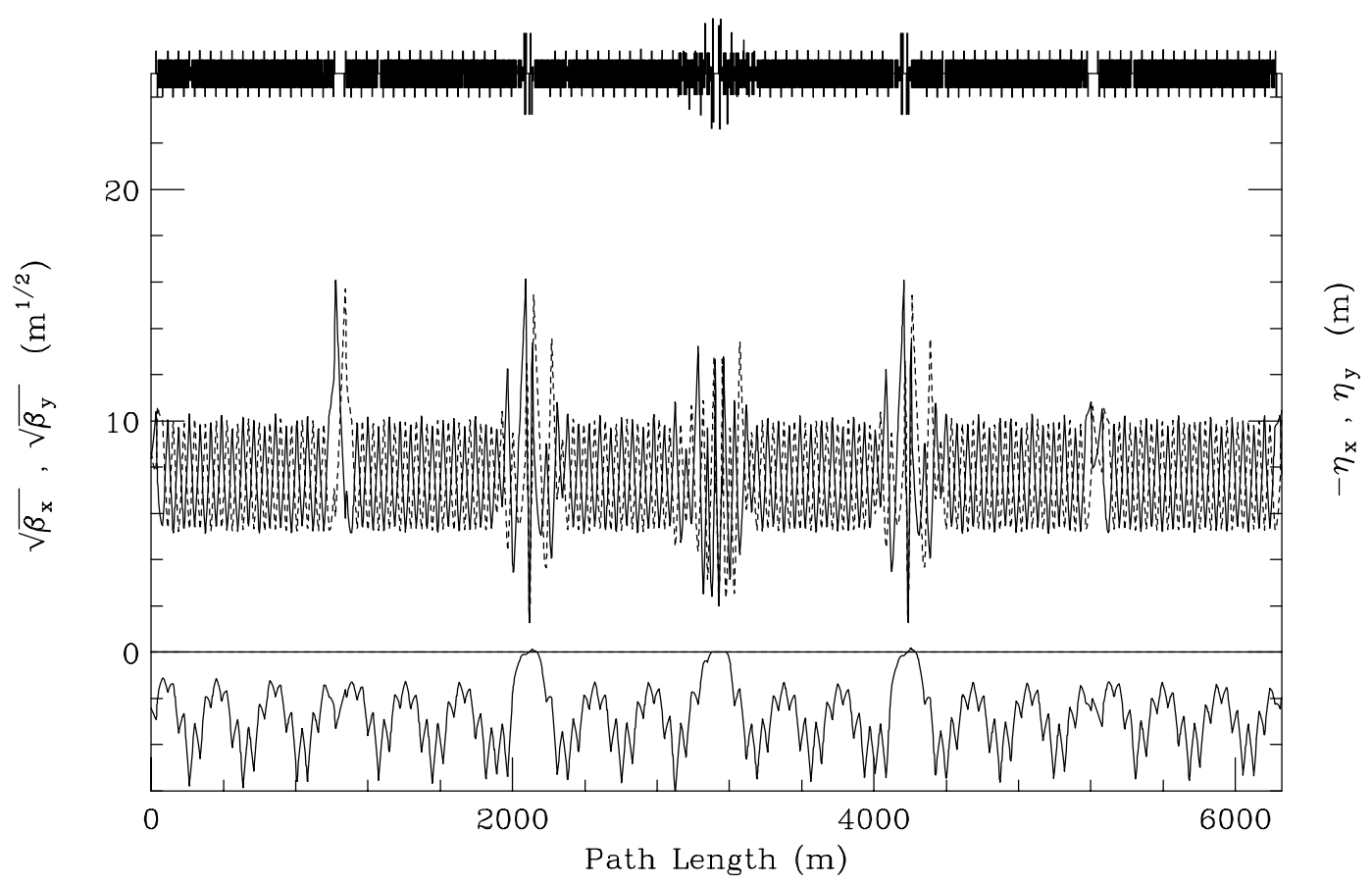

\begin{tabular}{||c||c|c||}
\hline \hline Quad \# & \multicolumn{2}{c||}{$\begin{array}{c}\text { B0 @ } \beta^{*}=4.00 \mathrm{~m} \\
\text { up }\left(\beta^{*}, \beta^{*} \mathrm{y}\right)=(1.61,1.74) \\
\text { down }\end{array}$} \\
\hline \hline Q1 & -159.197 & 159.197 \\
\hline Q2 & 182.773 & -182.773 \\
\hline Q3 & -180.026 & 180.026 \\
\hline Q4 & 165.415 & -165.415 \\
\hline QT5 & -5.380 & 5.380 \\
\hline & & \\
\hline QT6 & 61.267 & -50.421 \\
\hline QT7 & -116.421 & 110.525 \\
\hline QT8 & 0.192 & $\mathbf{2 2 . 0 9 8}$ \\
\hline QT9 & -12.545 & $\mathbf{- 1 6 . 5 7 2}$ \\
\hline QTA & 16.989 & \\
\hline QTB & -18.316 & \\
\hline
\end{tabular}




\subsubsection{2. $\beta^{*}=4.00 @ \mathrm{C} 0-\beta^{*}=0.35 @ \mathrm{~B} 0 \& \mathrm{D} 0$}

$\mathrm{C} 0 @ \beta^{*}=4.00 \mathrm{~m}: \mathrm{B} 0 \& \mathrm{D} 0 @ \beta^{*}=0.35 \mathrm{~m}$

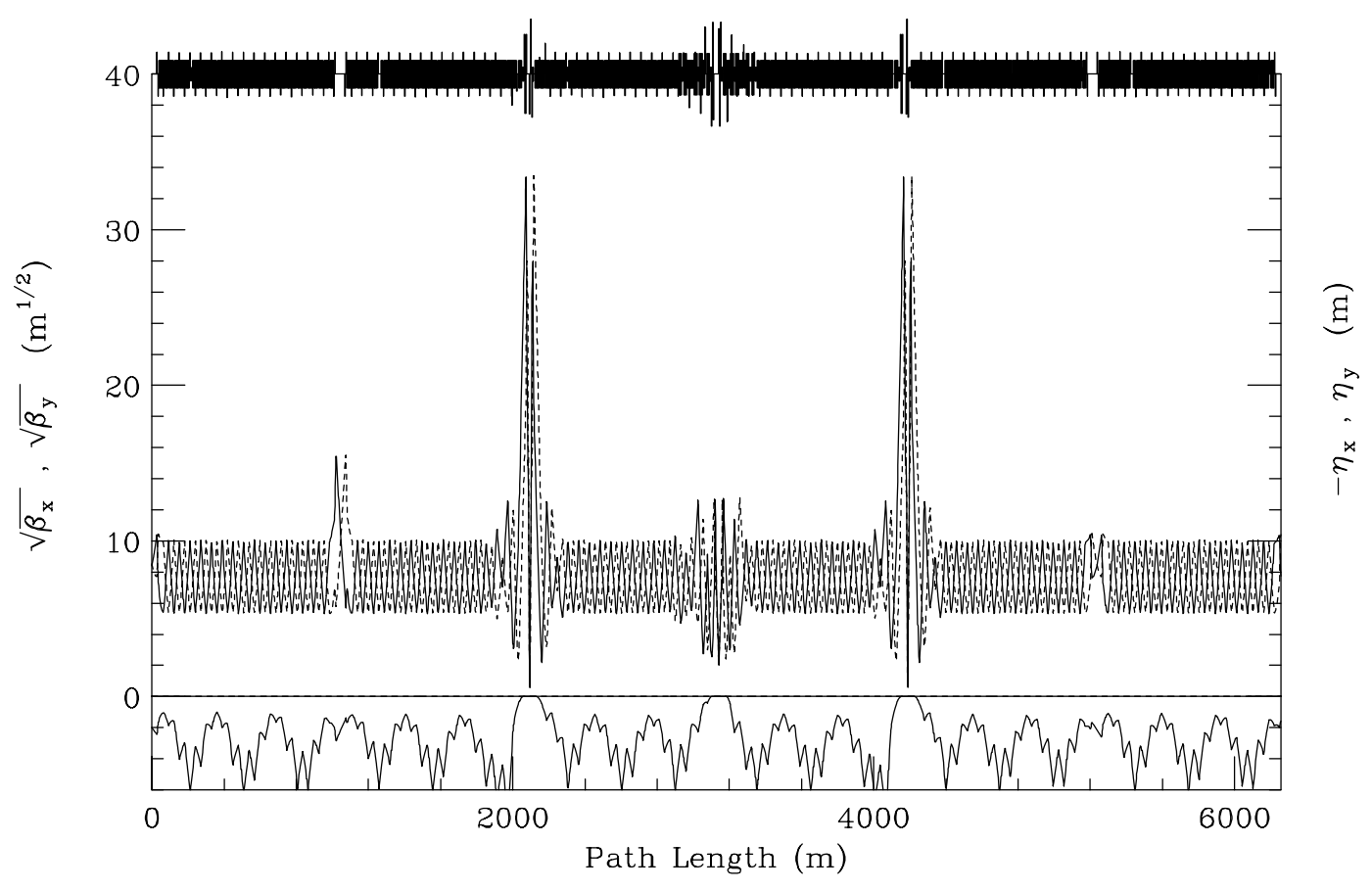

\begin{tabular}{||c||c|c||}
\hline \hline Quad \# & \multicolumn{2}{c||}{$\begin{array}{c}\text { C0 @ } \beta^{*}=4.00 \mathrm{~m} \\
\text { up \& D0 @ } \beta^{*}=0.35 \mathrm{~m} \\
\text { down }\end{array}$} \\
\hline \hline Q1 & -158.808 & 158.808 \\
\hline Q2 & 182.161 & -182.161 \\
\hline Q3 & -181.170 & 181.170 \\
\hline Q4 & 165.529 & -165.529 \\
\hline QT5 & -1.755 & 1.755 \\
\hline & & \\
\hline QT6 & 54.929 & -43.812 \\
\hline QT7 & -113.596 & 101.506 \\
\hline QT8 & 3.083 & $\mathbf{2 3 . 7 8 8}$ \\
\hline QT9 & -14.645 & $-\mathbf{1 3 . 4 8 4}$ \\
\hline QTA & 15.655 & \\
\hline QTB & -9.758 & \\
\hline
\end{tabular}

The 2 preceding tables listed C0 IR gradients corresponding, for example, to the endpoints of an operating scenario in which $\beta^{*}$ at $\mathrm{C} 0$ is fixed at $4.00 \mathrm{~m}$ while $\beta^{*}$ at B0 \& D0 is squeezed from the Injection values $\rightarrow \beta^{*}=0.35 \mathrm{~m}$ for collisions. At each step of this low- $\beta$ squeeze the C0 magnets are adjusted to maintain the optical match to the 'appropriate', ever-changing lattice functions \& phase advances across the insert. The following table indicates the extent to which these gradients vary during the B0 \& D0 squeeze. 


\begin{tabular}{|c|c|c|c|}
\hline & $\begin{array}{c}\mathrm{B}^{\prime}[\max ] \\
\mathrm{T} / \mathrm{m}\end{array}$ & $\begin{array}{c}\mathrm{B}^{\prime}[\min ] \\
\mathrm{T} / \mathrm{m}\end{array}$ & $\begin{array}{c}\Delta \int \mathrm{B}^{\prime} \bullet \mathrm{ds} \\
\mathrm{T}-\mathrm{m} / \mathrm{m}\end{array}$ \\
\hline Q1 & 159.505 & 158.893 & 1.44 \\
\hline Q2 & 183.764 & 182.767 & 3.97 \\
\hline Q3 & 180.667 & 179.156 & 3.57 \\
\hline Q4 & 159.547 & 158.745 & 1.35 \\
\hline QT5 & 1.381 & 0.347 & 0.66 \\
\hline & & & 3.26 \\
\hline QTB6 & 63.996 & 58.866 & 1.46 \\
\hline QTB7 & 117.517 & 115.112 & 1.72 \\
\hline QTB8 & 7.761 & 5.048 & 3.50 \\
\hline QTB9 & 13.138 & 7.625 & 1.70 \\
\hline QTBA & 15.779 & 13.099 & 4.60 \\
\hline QTBB & 17.448 & 10.205 & \\
\hline & & & 5.64 \\
\hline QTC6 & 60.315 & 51.426 & 4.83 \\
\hline QTC7 & 114.890 & 106.930 & 0.75 \\
\hline QTC8 & 9.449 & 8.265 & 2.86 \\
\hline QTC9 & 17.613 & 13.113 & \\
\hline
\end{tabular}

2.2.1.3. $\beta^{*}=0.50 @ \mathrm{C} 0-\left(\beta \mathrm{x}^{*}, \beta \mathrm{y}^{*}\right)=(1.61,1.74) @ \mathrm{~B} 0 \& \mathrm{D} 0$

C0 @ $\beta^{*}=0.50 \mathrm{~m}: \mathrm{BO} \& \mathrm{DO} @$ INJECTION

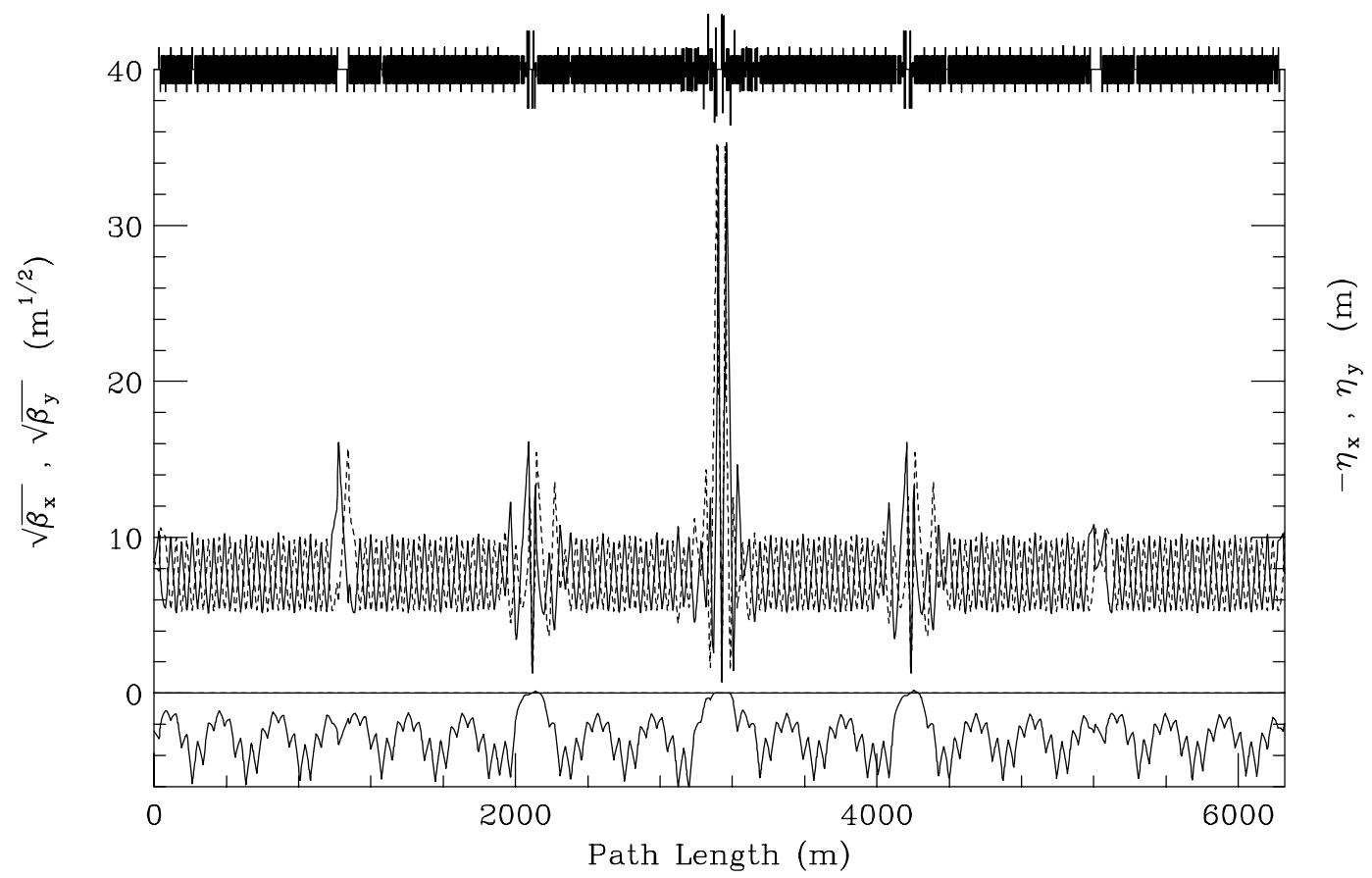




\begin{tabular}{||c||c|c||}
\hline \hline Quad \# & \multicolumn{2}{c||}{$\begin{array}{c}\text { C0 @ } \beta^{*}=0.50 \mathrm{~m} \\
\text { up }\left(\beta^{*}, \beta^{*}\right)=(1.61,1.74) \\
\text { down }\end{array}$} \\
\hline \hline Q1 & -159.787 & 159.787 \\
\hline Q2 & 179.807 & -179.807 \\
\hline Q3 & -180.416 & 180.416 \\
\hline Q4 & 191.400 & -191.400 \\
\hline QT5 & -52.248 & 52.248 \\
\hline & & \\
\hline QT6 & 2.849 & -6.700 \\
\hline QT7 & -67.307 & 80.573 \\
\hline QT8 & 8.930 & $-\mathbf{2 0 . 6 3 0}$ \\
\hline QT9 & -9.832 & $\mathbf{1 9 . 6 9 4}$ \\
\hline QTA & -1.100 & \\
\hline QTB & -12.828 & \\
\hline \hline
\end{tabular}

2.2.1.4. $\beta^{*}=0.50 @ \mathrm{C} 0-\beta^{*}=0.35 @ \mathrm{~B} 0 \& \mathrm{D} 0$

C0 @ $\beta^{*}=0.50 \mathrm{~m}: \mathrm{B} 0$ \& D0 @ $\beta^{*}=0.35 \mathrm{~m}$

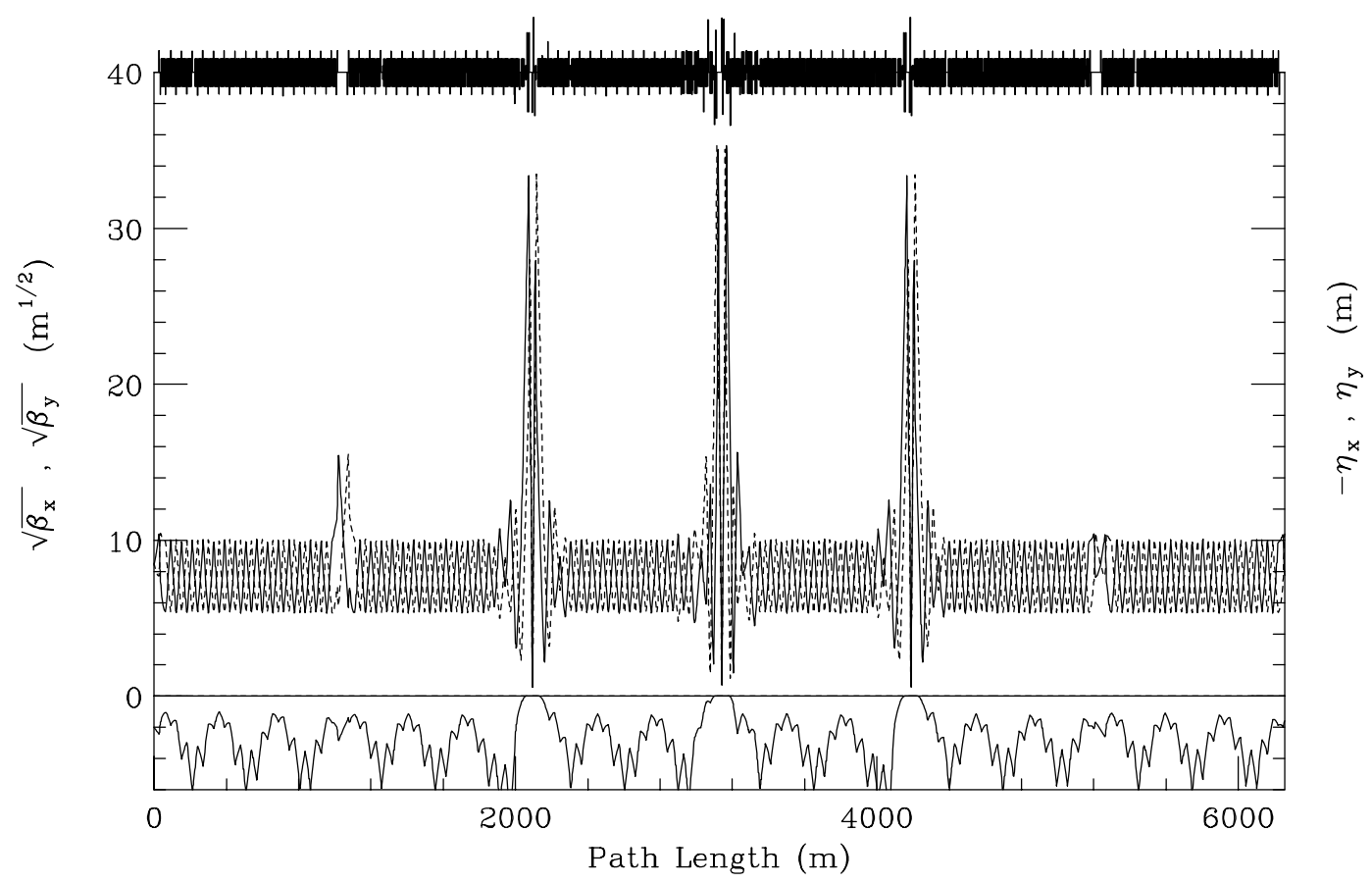




\begin{tabular}{||c||c|c||}
\hline Quad \# & \multicolumn{2}{c||}{$\begin{array}{c}\text { C0 @ } \beta^{*}=0.50 \mathrm{~m} \\
\text { up D0 @ } \beta^{*}=0.35 \mathrm{~m} \\
\text { down }\end{array}$} \\
\hline \hline Q1 & -160.126 & 160.126 \\
\hline Q2 & 180.398 & -180.398 \\
\hline Q3 & -180.909 & 180.909 \\
\hline Q4 & 184.500 & -184.500 \\
\hline QT5 & -45.193 & 45.193 \\
\hline & & 14.561 \\
\hline QT6 & -9.753 & 65.942 \\
\hline QT7 & -62.911 & $\mathbf{- 9 . 2 5 3}$ \\
\hline QT8 & 9.292 & $\mathbf{1 7 . 4 8 6}$ \\
\hline QT9 & -16.317 & \\
\hline QTA & -2.099 & \\
\hline QTB & -11.577 & \\
\hline \hline
\end{tabular}

With $\beta^{*}$ at B0 \& D0 fixed at $0.35 \mathrm{~m}$, the following graph shows the variation of the high-field quad gradients through a $\mathrm{C} 0$ squeeze from $\beta^{*}=4.00 \mathrm{~m} \rightarrow \beta^{*}=0.50 \mathrm{~m}$.

High-Field Quad Gradient Variations with $\beta^{*}$

[B0 \& D0 @ $\beta^{*}=0.35 \mathrm{~m}$ ]

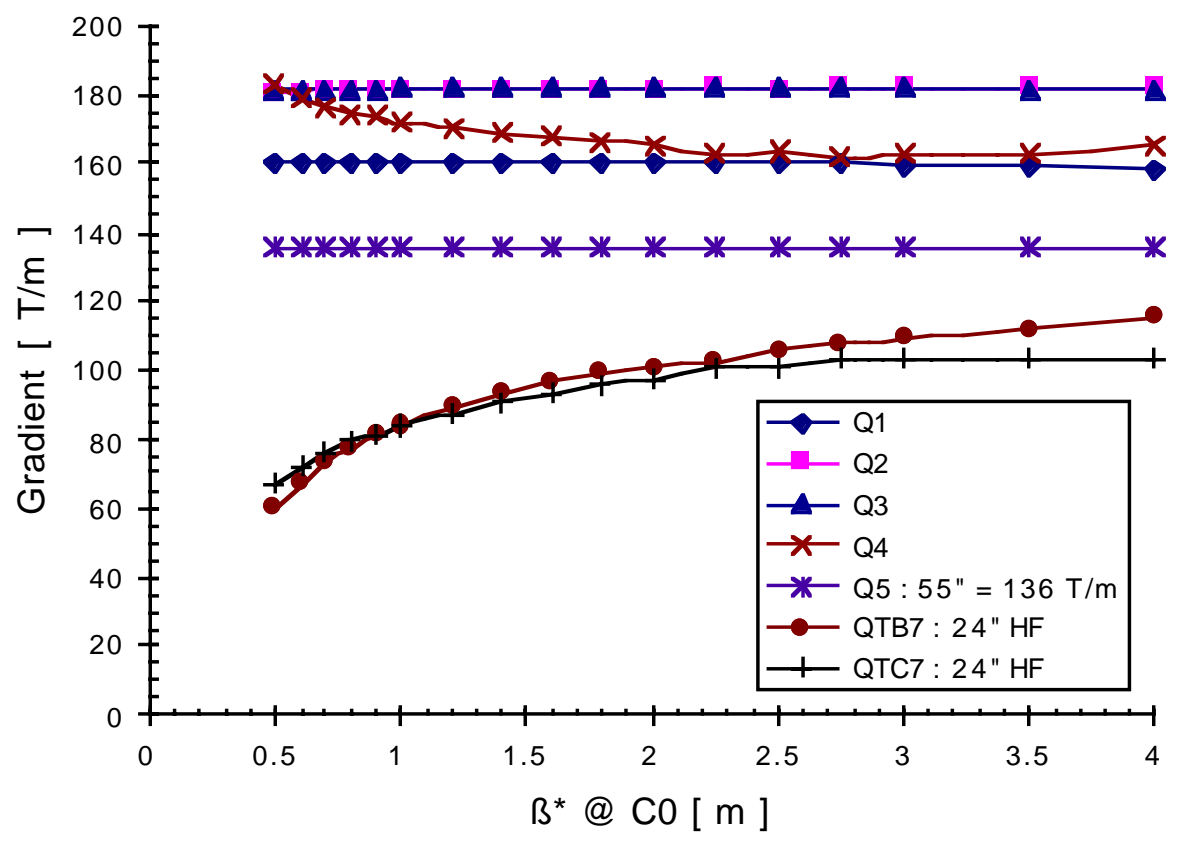


It is important that the new insertion has the optical versatility to match between all the conceivable combinations of $\beta^{*}$ at the 3 IR's. While it is imagined that all 3 IR's will operate at low- $\beta$ simultaneously, it is unlikely that they will be squeezed in tandem. It is well known that between each step of the B0 \& D0 low- $\beta$ squeeze a quadratic tune shift appears. By the sequential squeezing of B0 \& D0, followed by C0 (or vice versa), this problem isn't compounded unnecessarily.

\subsection{Beam Separation}

To reduce the number of interactions per crossing at the IP's, it is planned in Run IIb to reduce bunch spacing in the Tevatron from $396 \rightarrow 132$ nsec. With the first parasitic crossings then occurring just $19.86 \mathrm{~m}$ from the IP's, though, it is realized that crossing angles must be introduced to obtain separated beams at these points ${ }^{3}$.

Collider operation with crossing angles has at least 2 major consequences. First, luminosity is reduced due to the decreased overlap of the beams at the IP. A compromise must therefore be reached between minimizing the beam-beam tune shift from the first parasitic crossings (large $\theta_{1 / 2}$ ) $\&$ minimizing the luminosity reduction (small $\theta_{1 / 2}$ ). The second impact of crossing angles is to produce separated beams in the low- $\beta$ final-focus quadrupoles - precisely where $\beta$ already reaches its ring-wide maximum. With head-on collisions the Collider currently operates with $\beta^{*}=35 \mathrm{~cm}$, and $\beta_{\max }$ in the triplets is then $\sim 1100 \mathrm{~m}$. It is generally suspected (though not verified) that the minimum $\beta^{*}$ attainable is limited by the adverse impact on the beam by high-order multipoles in the low- $\beta$ quadrupoles. The consequences of sending beams off-axis through the magnets as well is not well understood - the Tevatron has never been operated before with crossing angles.

Beyond the decisions to implement shorter bunch spacing \& crossing angles in Run IIb, many details of collision scenarios have not been finalized. Significantly, for instance, there is no 'official' helix to describe Collider operations in this era - several promising candidates exist - and the final locations of separators have not yet been established. The collision helix assumed for the purposes of the current study should be viewed, therefore, as only the latest helix du jour, and might be quite different in detail from the eventual configuration. In this version 4 the half-crossing angles at B0 \& D0 are $\left(\mathrm{x}^{\prime *}, \mathrm{y}^{\prime *}\right)=(+170,-170) \mu \mathrm{rad}$, giving $5 \sigma$ of separation at the $1^{\text {st }}$ crossing for $\beta^{*}=35 \mathrm{~cm}$, and $20 \pi$ emittance $(95 \%$, normalized) beams.

This particular incarnation of the Run IIb helix, unfortunately, is not well-suited for also supporting collisions at $\mathrm{C} 0$. Beam separation, with the standard Collins insert at $\mathrm{C} 0$ still installed, is shown in the following figures. Separation is clearly poorest in the short B0 $\rightarrow \mathrm{C} 0 \rightarrow \mathrm{D} 0$ arc, dipping near to $5 \sigma$ in several locations. Furthermore, the illustration of just the $\mathrm{B} 38 \rightarrow \mathrm{C} 21$ section suggests it will be difficult for a new IR insertion to create comfortable beam separation in the vicinities of the minima at $\mathrm{B} 47$ \& $\mathrm{C} 13$, while maintaining the requisite match to the nominal helix.

New arc separators in this model have been situated to optimize beam separation, consistent with this one, specific, Run IIb helix solution. With separators at B43, B46, C13, and C16, collisions can be created at all 3 IP's while matching to the nominal incoming \& outgoing helices across the $\mathrm{C} 0$ insert. (It is also possible to have collisions at just B0 \& D0, or just C0, of course. Discussion of these less interesting operational modes is postponed, however, until the next section where, in the $2^{\text {nd }}$ IR version, these become the only operational choices).

$3132 \mathrm{nsec}$ Bunch Spacing in the Tevatron Proton-Antiproton Collider, S.D. Holmes, et al., TM-1920.

4 "v3h15acsb4.nppn.170pnpn", Peter Bagley, private communication. 
Run II Separation with CO Collins Insert
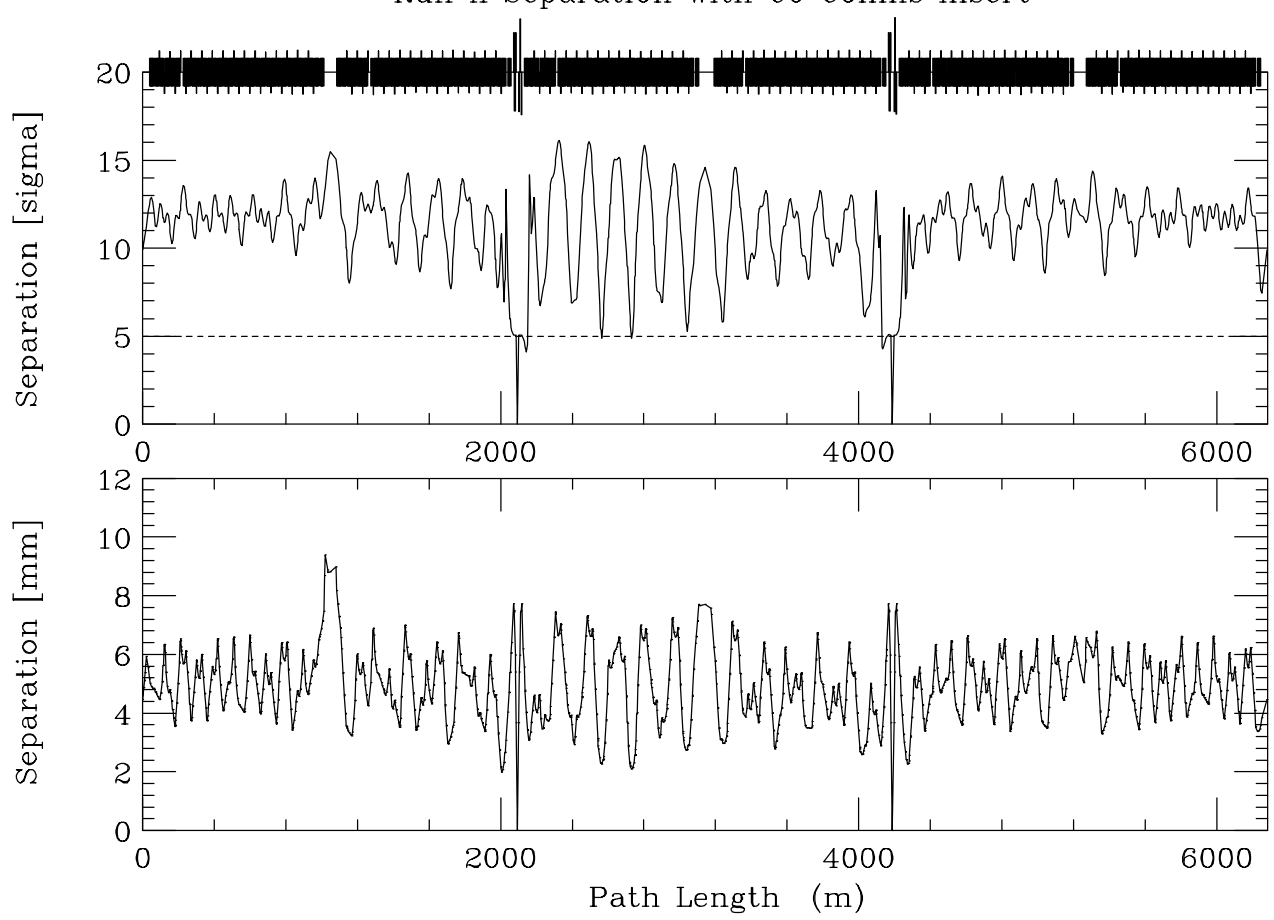

Run II B38 -> C21 with Collins Straight

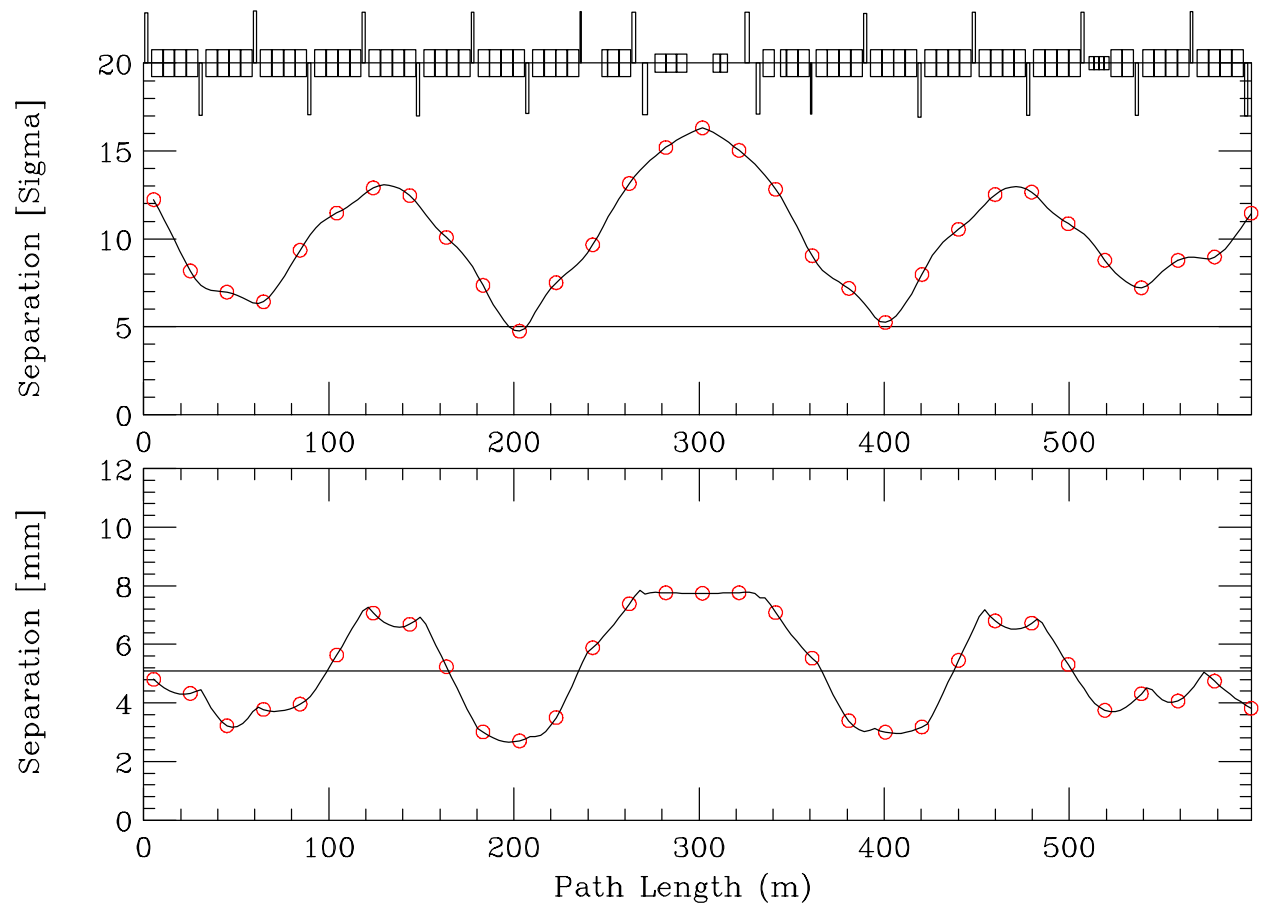


Shown below is the beam separation from B38 $\rightarrow$ C21. At the IP, $\beta^{*}=0.50 \mathrm{~m}$ and there are halfcrossing angles of $\left(\mathrm{x}^{\prime *}, \mathrm{y}^{\prime *}\right)=(-195,+195) \mu \mathrm{rad}$, giving $7 \sigma$ separation at the first parasitic crossings. Other potential collision points are indicated, spaced at 7 half-bucket intervals. The separation is generally acceptable but, as anticipated, is poorest each side of the IP - hovering near $5 \sigma$ until outboard of the 6th secondary crossing points around B46 \& C14.

Collisions at BO, CO, \& DO
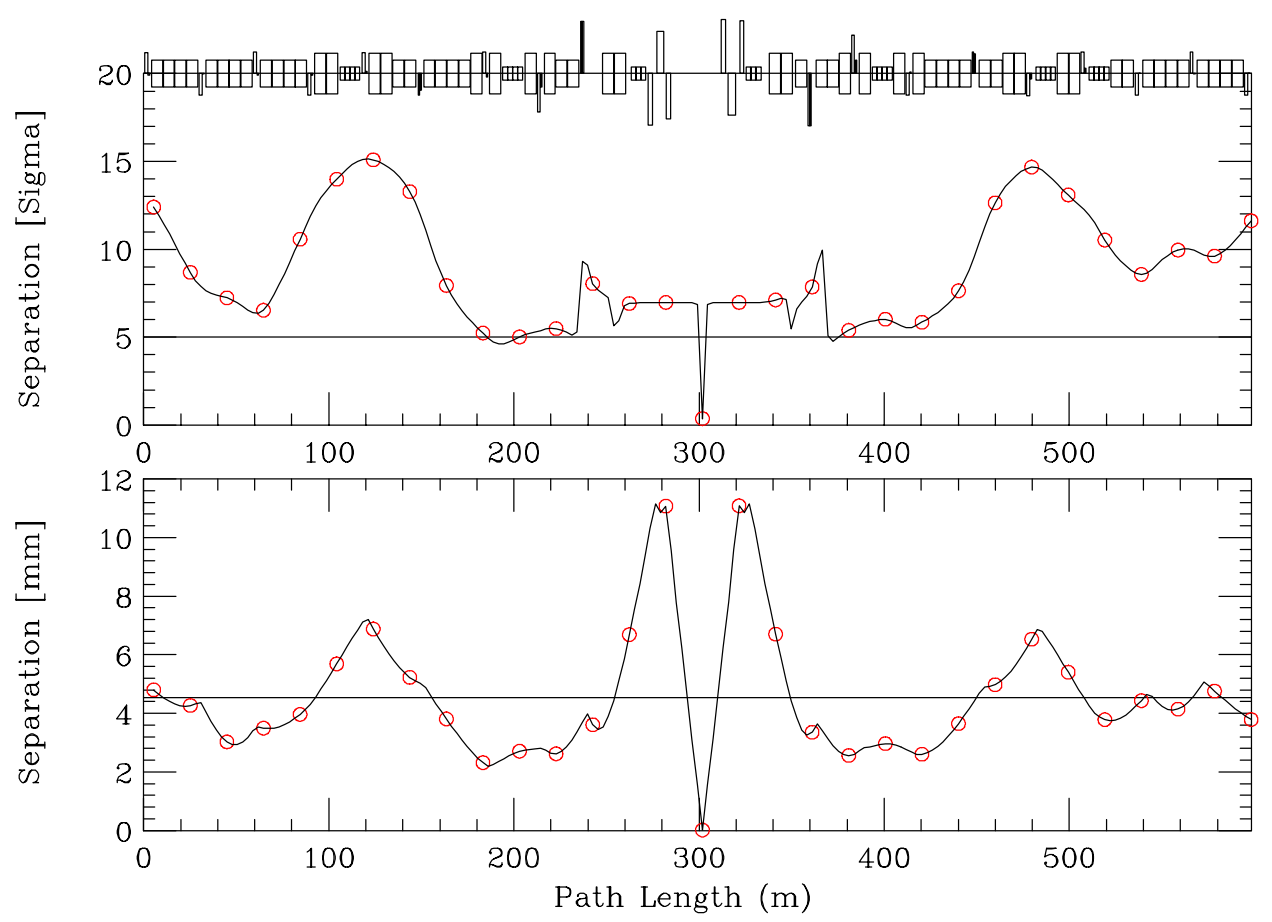

The large crossing angles ( $275 \mu$ rad total half-angle) are necessary to keep the beams adequately separated through the natural minima of the Run IIb helix. However, the impact on luminosity is at least no worse here than the crossing angle effect at B0 \& D0. A crossing angle reduces the luminosity relative to that of head-on collisions, $\mathrm{L}_{\mathrm{O}}$, by the factor:

$$
\mathrm{L}_{\theta}=\left[1+\left(\theta_{1 / 2} \cdot \sigma_{1} / \sigma_{\perp}\right)^{2}\right]^{-1 / 2} \cdot \mathrm{L}_{0}
$$

where $\theta_{1 / 2}$ is the total half-crossing angle, $\sigma_{1}$ is the rms bunch length, and $\sigma_{\perp}$ is the rms transverse bunch size. For the purposes of the present discussion the relevant quantity is the transverse ratio $\theta_{1 / 2} / \sigma_{\perp}$. For "N" $\sigma$ of separation at the first crossing this has the approximate value:

$$
\frac{\theta_{1 / 2}}{\sigma_{\perp}} \approx \frac{\mathrm{N}}{2 \beta^{*}}
$$

A $\beta^{*}$ of $50 \mathrm{~cm}$ and $7 \sigma$ separation at the $1^{\text {st }}$ crossing, therefore, has an equivalent impact on luminosity as $5 \sigma$ separation with $\beta^{*}=35 \mathrm{~cm}$.

Separator gradients for this solution are listed in the table below, followed by the beam separation through the short arc B0 $\rightarrow \mathrm{C} 0 \rightarrow \mathrm{D} 0$. The very small gradients at B49 horizontally, and $\mathrm{C} 11$ vertically, are a further indication that the nominal helix is not a 'natural' candidate for $\mathrm{C} 0$ collision scenarios. One would prefer to see large kicks at these locations to initiate separation into the arcs. 


\begin{tabular}{||c|c|c|c|c|c||}
\hline \multicolumn{7}{||c|}{$\begin{array}{c}\text { Separator Gradients } \\
(\mathrm{MV} / \mathrm{m})\end{array}$} \\
\hline & & & $\mathrm{B} 43$ & 4 & -2.14759 \\
\hline B46 & 4 & 3.00372 & & & \\
\hline B49 & 2 & -0.48634 & B49 & 1 & -2.93754 \\
\hline C11 & 1 & -3.24089 & C11 & 2 & 0.60083 \\
\hline & & & C13 & 4 & 3.34755 \\
\hline C16 & 4 & -1.75608 & & & \\
\hline
\end{tabular}
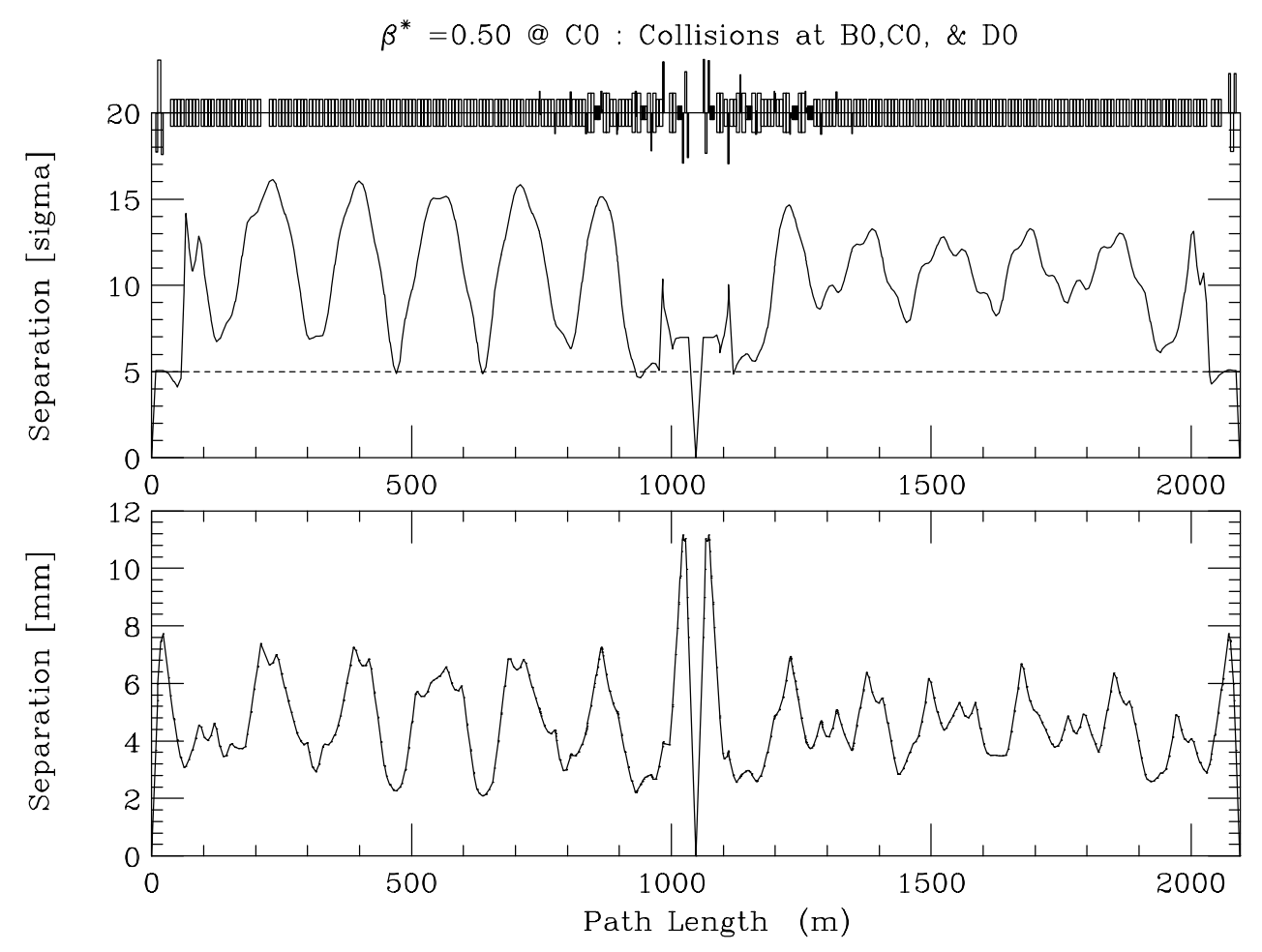

\section{AN IR With STANDARd TEVATRON DipoleS}

\subsection{Physical Design}

\subsubsection{Quadrupoles}

The IR quadrupole circuits, indicated on the following page, are identical to those described in the preceding section.

\subsubsection{Dipoles \& Separators}

Having only standard Tevatron arc dipoles available for modelling has 2 significant consequences. First, it is not possible to add more separators in the arcs. Collisions at $\mathrm{C} 0$ can not be created without disrupting the nominal Run IIb helix outside of the insertion region. Second, the free space available for a detector shrinks markedly - by roughly $5 \frac{1}{2} 2 \mathrm{~m}$ relative to the previous design - to just $33^{1 / 2}$ feet each side of the IP. 


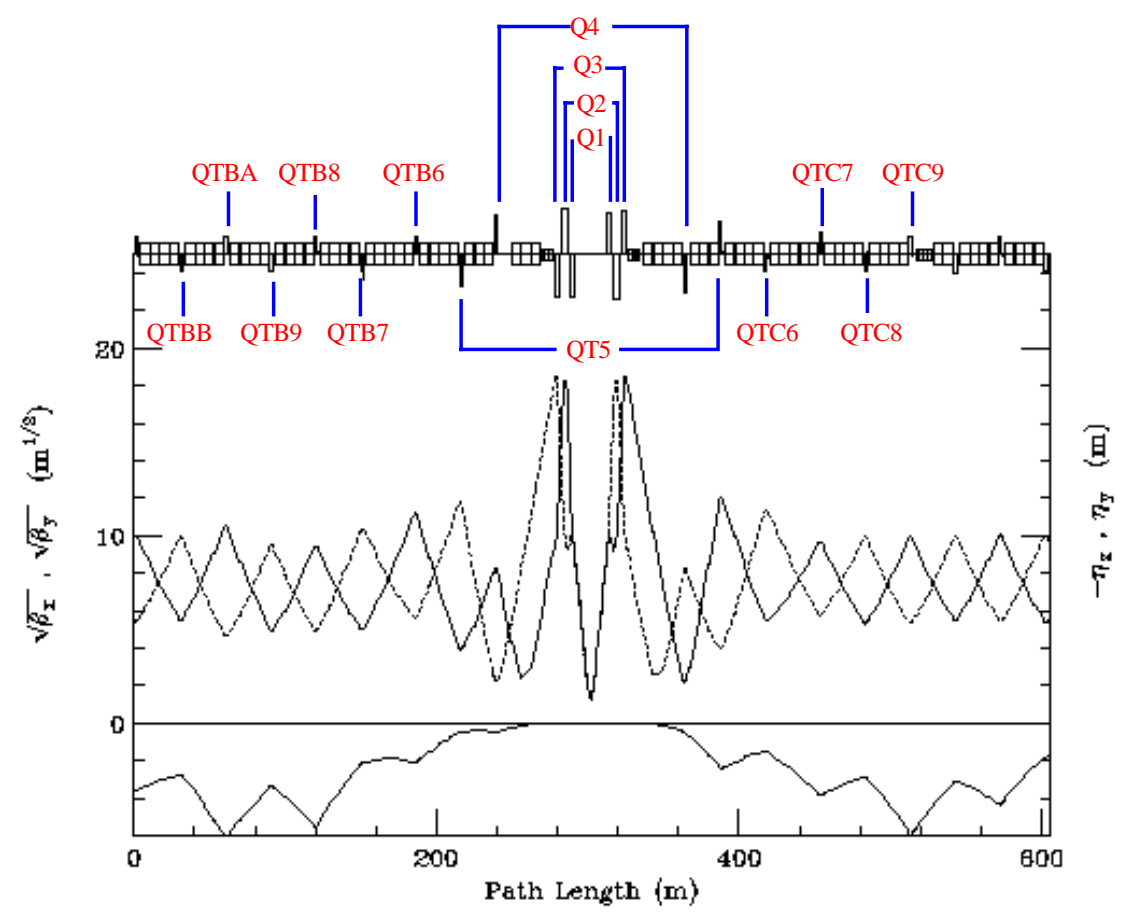

\subsection{Optics}

The injection \& collision optics are illustrated in the next 2 graphs. At injection, $\beta^{*}=2.80 \mathrm{~m}$ results in a $\beta$ max of $175 \mathrm{~m}$ in the triplets. Again, this is much less than at B0 \& D0. With the triplet quads closer to the IP in this version of the insert, a $\beta^{*}$ as small as $40 \mathrm{~cm}$ can be reached before $\beta \max$ becomes excessive. In all other respects the optical properties of this version of the IR are almost indistinguishable from those discussed in the preceding section. It would be odious, therefore, to present reams of nearly identical material again here.

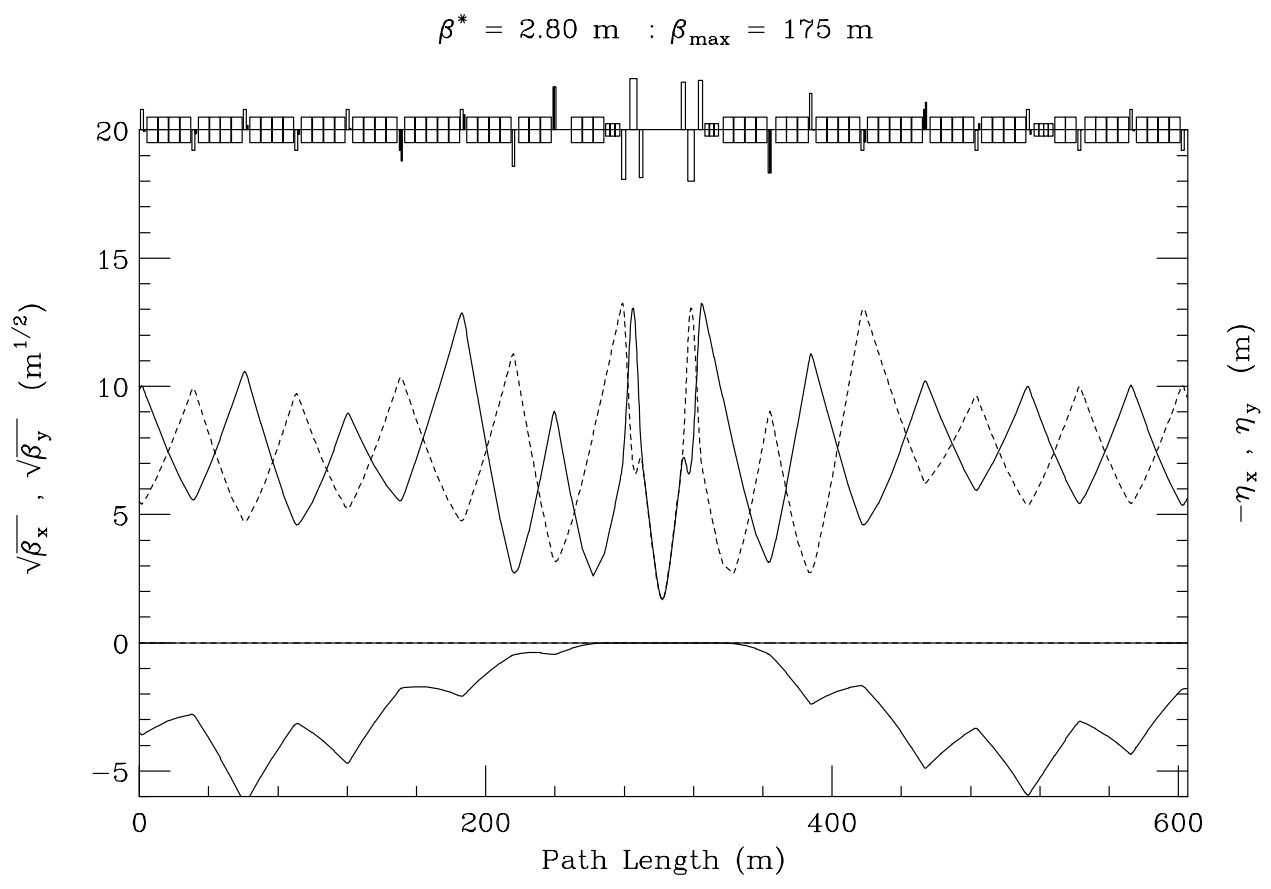




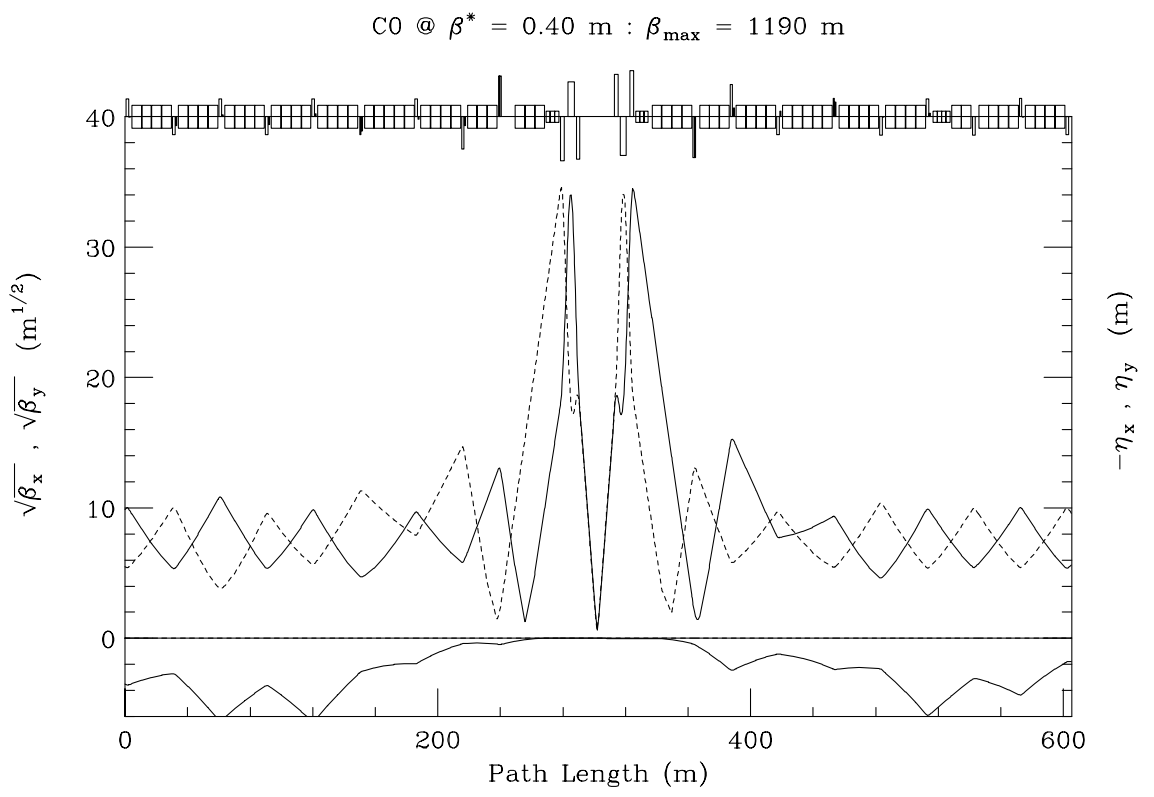

\subsection{Beam Separation}

With the restriction that $\mathrm{C} 0$ operations must not impact nominal Run IIb B0 \& D0 collider parameters, and without additional separators, the Tevatron Collider can operate in just 2 modes:

(1) B0 \& D0 with collisions - not C0, and ;

(2) C0 with collisions - not B0 \& D0.

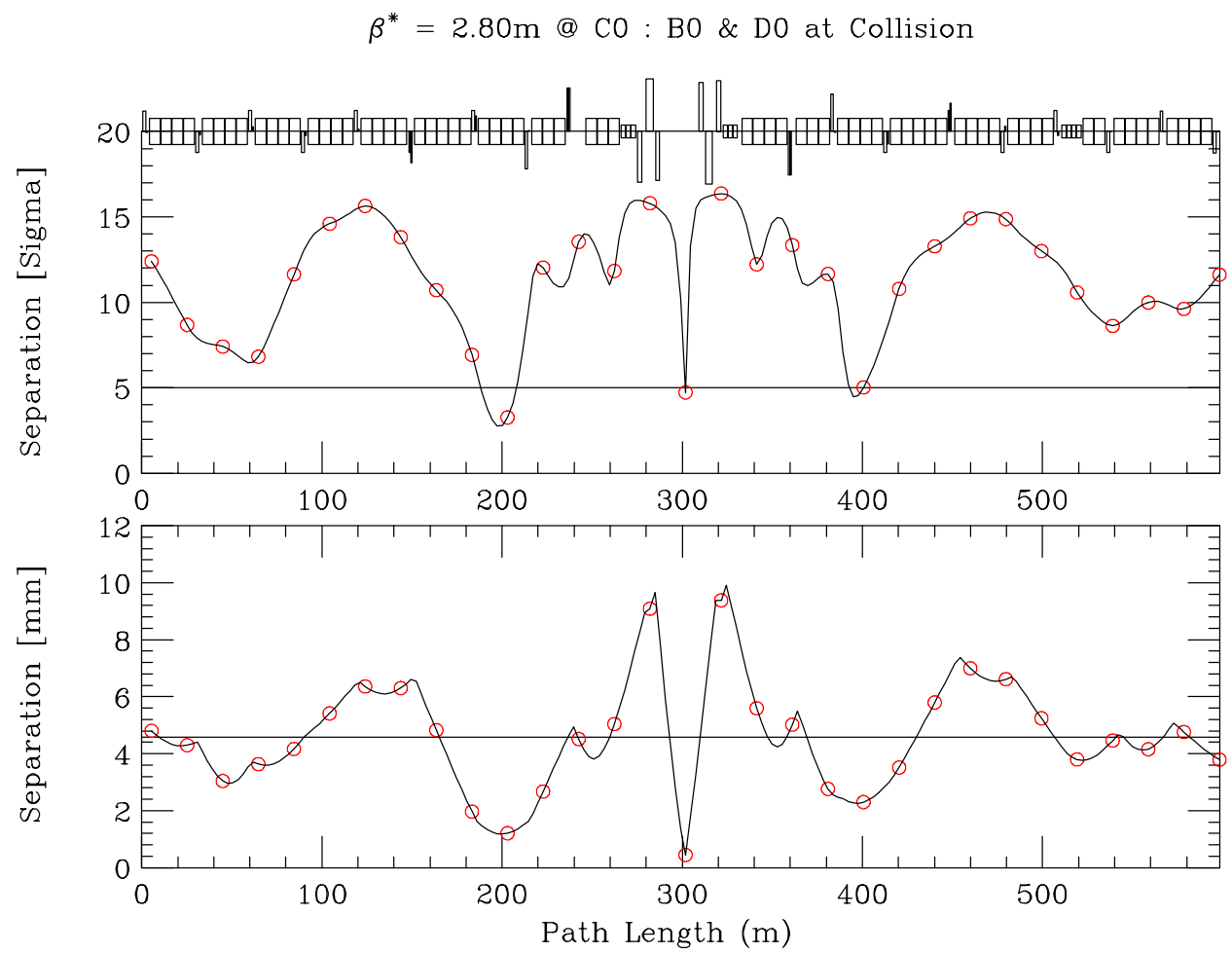




\subsubsection{Co Without Collisions}

Without collisions at $\mathrm{C} 0$ the insertion optics are fixed at $\beta^{*}=2.80 \mathrm{~m}$, and the $\mathrm{B} 49 \& \mathrm{C} 11$ separators are turned off. The resulting matched helix from B38 $\rightarrow \mathrm{C} 21$ is shown on the preceding page. Beam separation is $\geq 5 \sigma$ everywhere except for the 5 th crossing point on B-Sector side. There is really nothing that can be done locally to influence this point - it is largely a feature of the nominal Run IIb helix.

\subsubsection{Co With Collisions}

For collisions at C0 the optics at B0 \& D0 remain in their Injection configuration. In this case, all the separators in the ring become available for bringing beams together at the C0 IP, while keeping them separated everywhere else. For half-crossing angles at $\mathrm{C} 0$ of $\left(\mathrm{x}^{\prime *}, \mathrm{y}^{\prime *}\right)=(-180,+180) \mu \mathrm{rad}$, one possible (minimal) separator solution is given in the table below. The selection of separators has not been optimized in any way, other than to ensure adequate beam separation around the ring. Many, many more combinations can be explored.

\begin{tabular}{||c|c|c|c|c|c||}
\hline \multicolumn{7}{|c||}{$\begin{array}{c}\text { Separator Gradients } \\
(\mathrm{MV} / \mathrm{m})\end{array}$} \\
\hline & & & $\mathrm{B} 11$ & 1 & -2.35261 \\
\hline B17 & 4 & -0.102614 & & & \\
\hline B49 & 2 & -4.50000 & $\mathrm{~B} 49$ & 1 & 4.50000 \\
\hline C11 & 1 & 4.50000 & $\mathrm{C} 11$ & 2 & -4.50000 \\
\hline & & & $\mathrm{C} 17$ & 4 & -0.62098 \\
\hline C49 & 1 & -1.35185 & & & \\
\hline
\end{tabular}
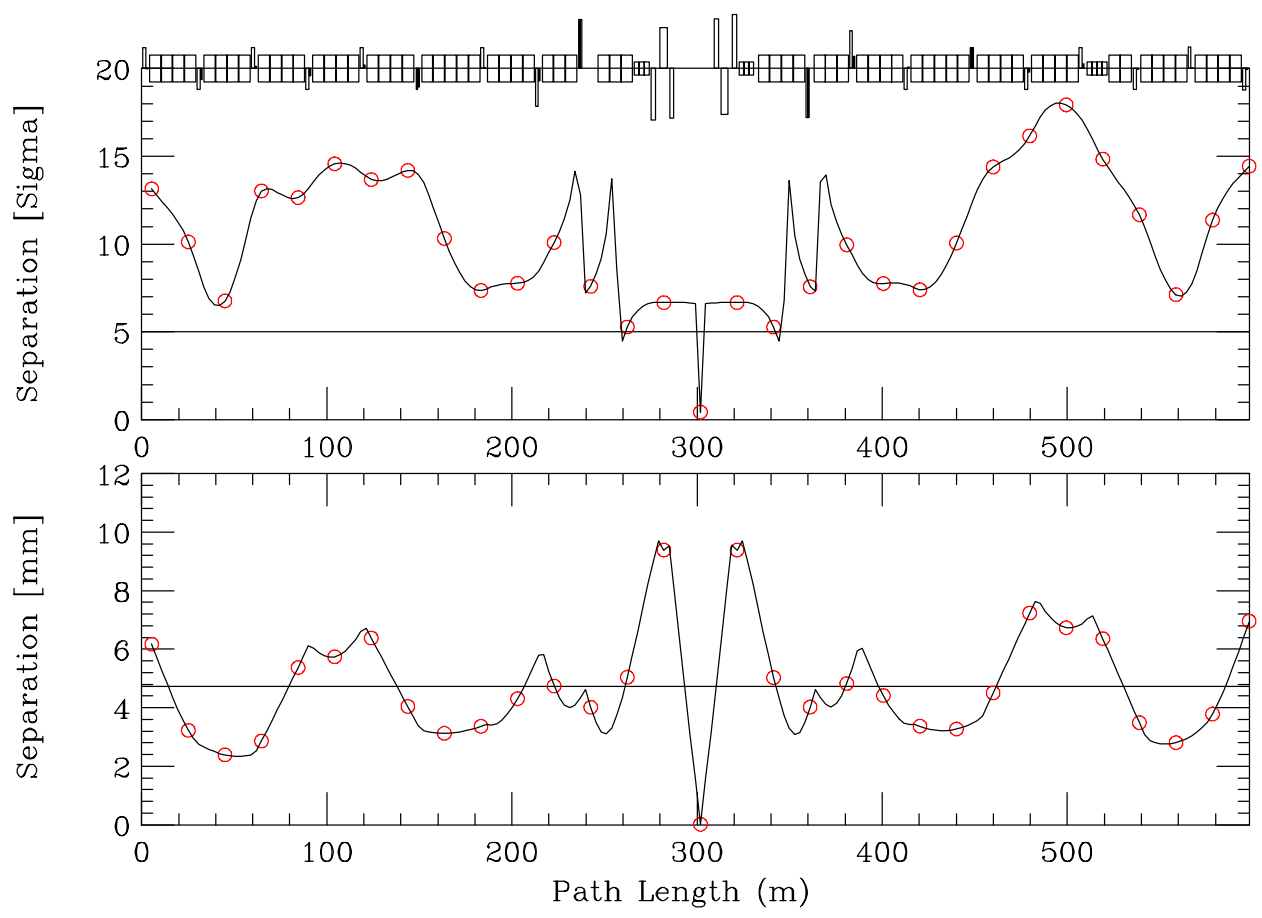


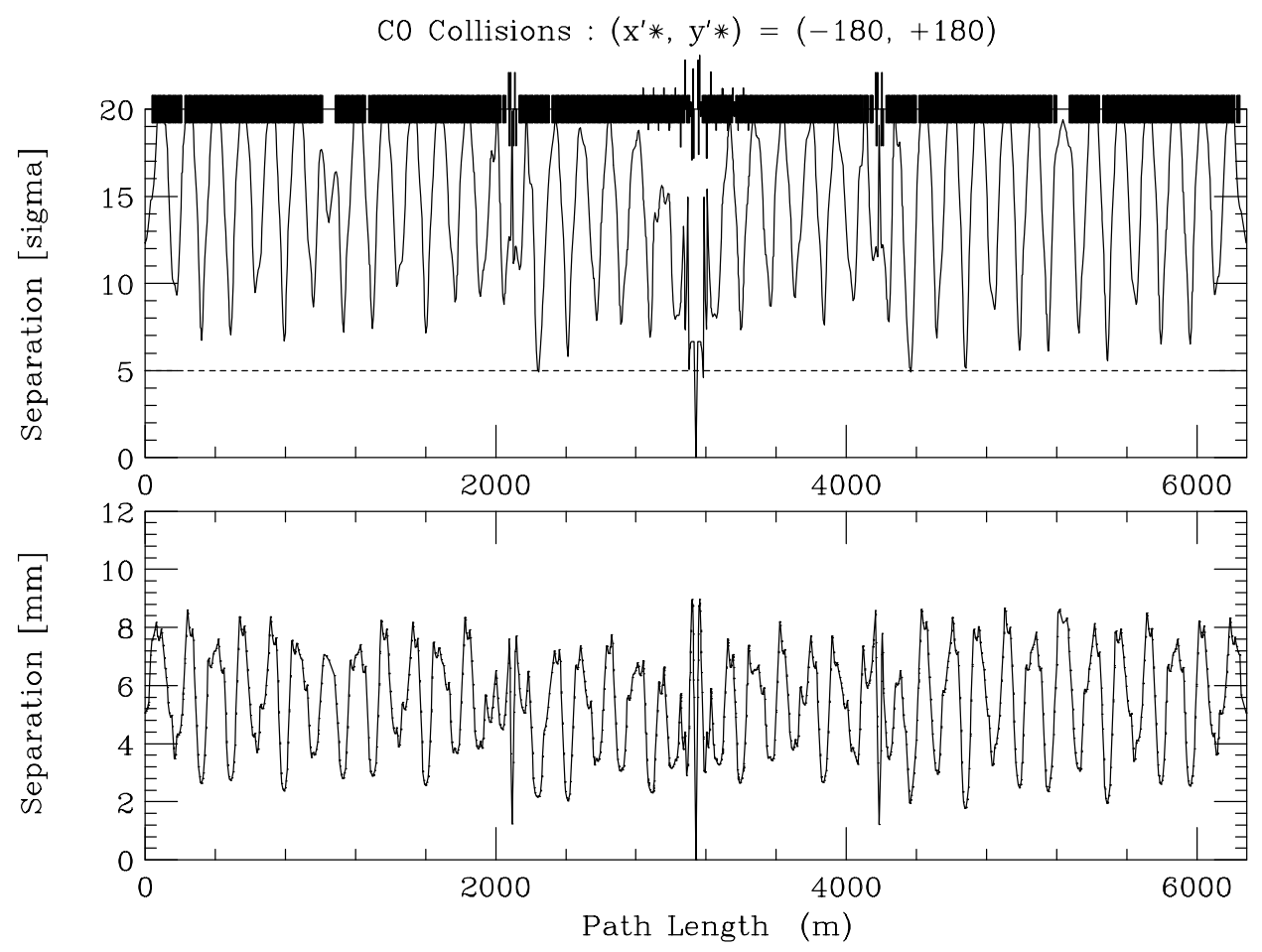

The preceding diagrams showed the beam separation from $\mathrm{B} 38 \rightarrow \mathrm{C} 21$, and the separation all around the ring. With this separator solution the closest approach through the insert is at the $3^{\text {rd }}$ parasitic crossing but, nonetheless, it is still $\geq 5 \sigma$ everywhere. Similarly, separation in the ring drops close to $5 \sigma$ in a few spots, but the average separation is $10 \rightarrow 15 \sigma$. The use of a larger subset of separators would enable the large oscillations to be smoothed from the helix.

\subsection{Collisions at BO, C0, \& Do}

In this version of the IR model, collisions can not occur at all 3 IP's consistent with the C0 insert remaining optically transparent. This section examines briefly, though, whether a reasonable 'semi-local' solution exists using only the separators in the short arc. There are 5 sets of separators, including the B49 \& C11 modules, in each plane between B0 and D0. With the B0 \& D0 crossing angles fixed at $\left(\mathrm{x}^{\prime *}, \mathrm{y}^{\prime *}\right)=(+170,-170) \mu \mathrm{rad}$, and $\left(\mathrm{x}^{\prime *}, \mathrm{y}^{\prime *}\right)=(-180,+180) \mu \mathrm{rad}$ at $\mathrm{C} 0$, only one separator solution exists with physically realizable gradients. Beam separation through the $\mathrm{C} 0$ insert \& corresponding separator gradients for this case are shown below.

\begin{tabular}{||c|c|c|c|c|c||}
\hline \multicolumn{7}{|c||}{$\begin{array}{c}\text { Separator Gradients } \\
\text { ( MV / m ) }\end{array}$} \\
\hline B11 & 1 & 1.55253 & B11 & 2 & -2.93408 \\
\hline B17 & 4 & -4.50000 & & & \\
\hline B49 & 2 & 2.55308 & B49 & 1 & -2.94817 \\
\hline C11 & 1 & -2.28540 & C11 & 2 & -4.50000 \\
\hline & & & C17 & 4 & 3.57661 \\
\hline C49 & 2 & -2.35765 & C49 & 1 & -2.30501 \\
\hline
\end{tabular}


Collisions at $\mathrm{BO}, \mathrm{CO}, \& \mathrm{DO}$

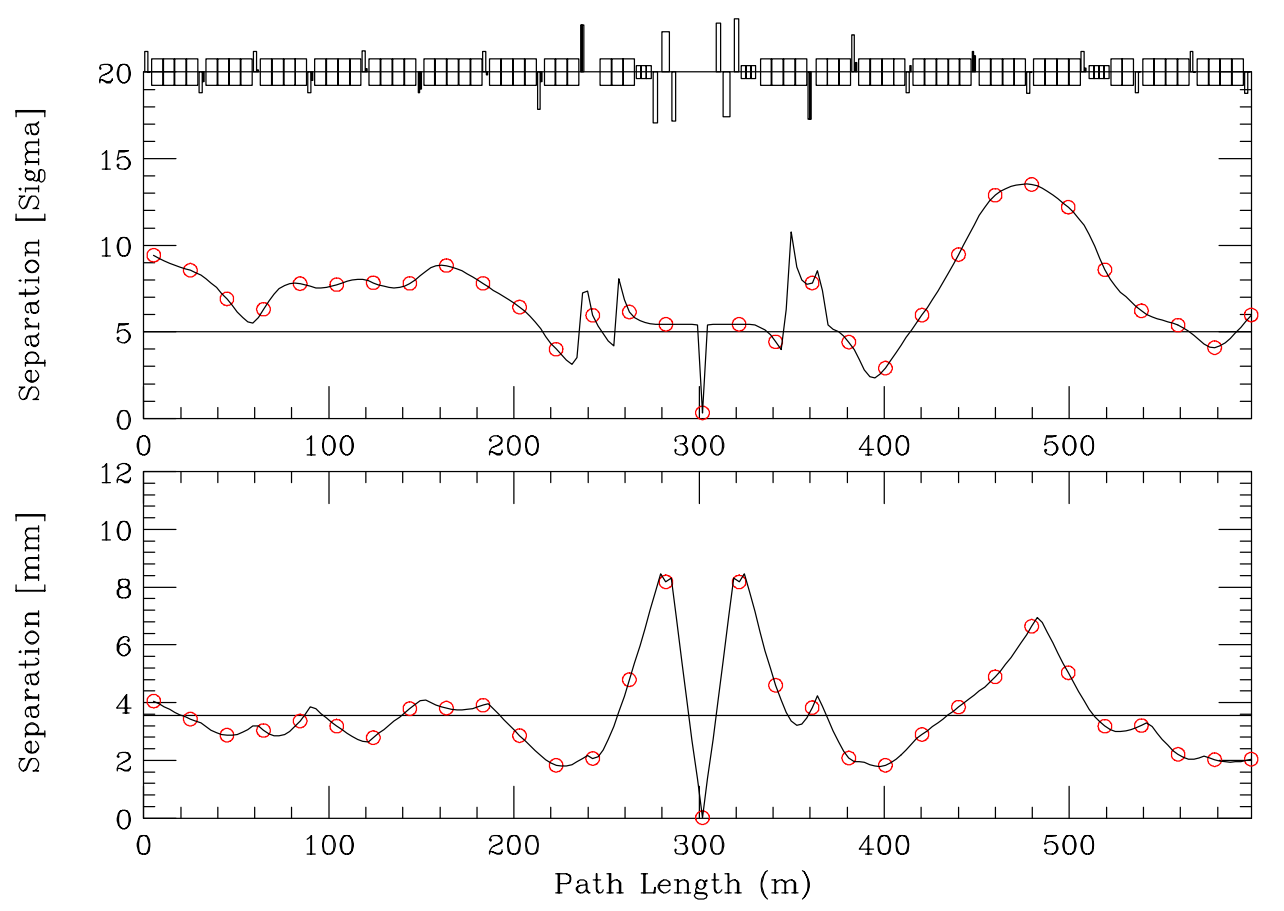

Splitting the crossing angle equally between the transverse planes optimizes beam separation into the arcs, but the solution obtained is clearly a very marginal situation. At several near-approach points through the insertion region beam separation drops below $5 \sigma$. The viability of this solution becomes even more suspect when separation in the short arc is compared with the rest of the ring (below). In going from $\mathrm{B} 0 \rightarrow \mathrm{C} 0 \rightarrow \mathrm{D} 0$ the separation is often less than $5 \sigma$, and average separation is only half that of the long arc $\mathrm{D} 0 \rightarrow \mathrm{E} 0 \rightarrow \mathrm{F} 0 \rightarrow \mathrm{A} 0 \rightarrow \mathrm{B} 0$.

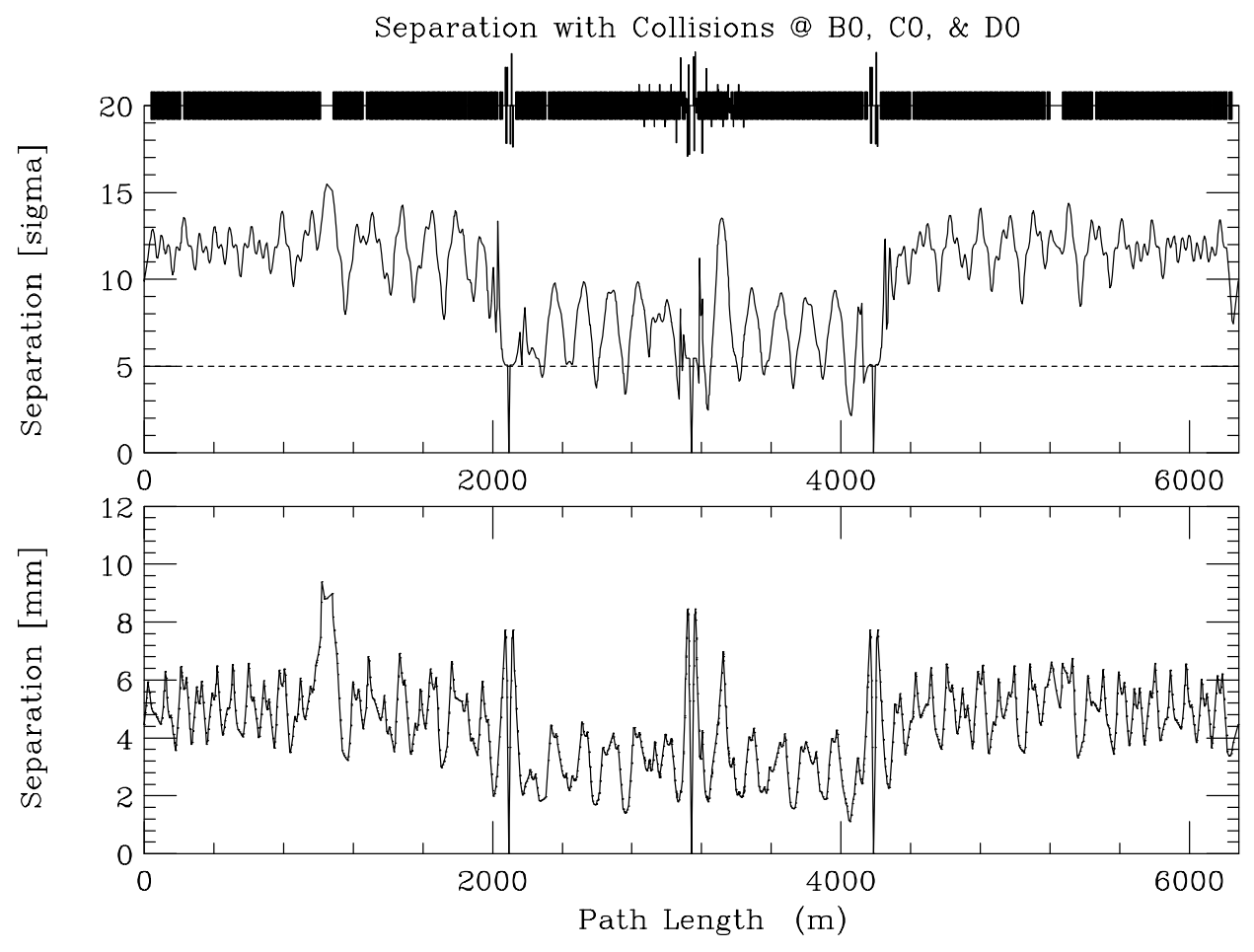




\section{Summary \& Observations}

Two possible conceptual optical designs for a stand-alone C0 IR insert were presented. Both inserts are optically transparent to the rest of the machine, with no impact on Run IIb Tevatron operating parameters. Both design variations require high-field LHC-like quadrupoles for the final focus triplet. In the first version, with enhanced dipoles creating space for separators in the arcs, collisions can be created at all 3 IP's simultaneously. Stronger dipoles also free more than $26 \mathrm{~m}$ of space for the detector. At $\mathrm{C} 0, \beta^{*}$ is limited to $\geq 50 \mathrm{~cm}$ by $\beta \max$ in the IR triplets. The second version of the IR has neither new dipoles nor new arc separators. Collider scenarios have either B0 \& D0 at collision, or just $\mathrm{C} 0$. At $\mathrm{C} 0, \beta^{*}$ can be decreased to $40 \mathrm{~cm}$, but the price paid is a substantial reduction in free space available for the detector.

This first pass at C0 IR designs has left a number of questions unresolved. A few of these outstanding issues that a second iteration of the IR designs must address are discussed below.

- The LHC-like quadrupoles operate with gradients somewhat higher than the present Tevatron cryogenics can tolerate. Reducing the strengths will require that the magnets become longer. If gradients are kept $\leq 175 \mathrm{~T} / \mathrm{m}$, the magnetic lengths of the triplet quads Q1, Q2, \& Q3 are estimated to grow by $\sim 5 \%$, and Q4 by $\sim 10 \%$, for a total of $\approx 24 "$. Both IR versions discussed here can easily accommodate these changes without encroaching on the detector space. In the IR model with enhanced dipoles a total of $17^{\prime}$ of free space currently exists between the downstream separators \& Q4. The design with only standard Tevatron dipoles has $51 / 4^{\prime}$ between the separators \& first dipole. In the model at least, length changes become just a technical detail.

- The addition of a new low- $\beta$ has a huge impact on chromaticity, changing the natural chromaticity of the machine by $\left(\Delta v_{\mathrm{X}}, \Delta v_{\mathrm{y}}\right)=(-19.75,-19.70)$. If the insertion is to be truly transparent a local sextupole correction scheme must be devised for compensation.

- With all 3 IP's at collision beam separation through the C0 IR is not as large as desired. Partly this is a consequence of the current Run IIb helix not being optimized in any way to account for $\mathrm{C} 0$ interactions. A comparison of separation through the insert with $\mathrm{B} 0, \mathrm{C} 0$, \& $\mathrm{D} 0$ collisions (section 2.3), with just $\mathrm{C} 0$ collisions (section 3.3.2), shows the large improvement in beam separation that can result with a different incoming helix. The options for alternative, global, separator solutions that optimize the collision helix for B0 \& D0, plus C0, should be explored.

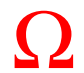

